\title{
Nonsimilar Modeling and Numerical Simulations of Electromagnetic Radiative Flow of Nanofluid with Entropy Generation
}

\author{
Muzamil Hussain $\mathbb{D}^{1,5}$ Jifeng Cui, ${ }^{2}$ Umer Farooq $\mathbb{D}^{1},{ }^{1}$ Mohammed Elamin Ahmed Rabie, ${ }^{3}$ \\ and Taseer Muhammad $\mathbb{1}^{4}$ \\ ${ }^{1}$ Department of Mathematics, COMSATS University Islamabad, Park Road Chak Shahzad, Islamabad 44000, Pakistan \\ ${ }^{2}$ College of Science, Inner Mongolia University of Technology, Hohhot 010051, China \\ ${ }^{3}$ Department of Mathematics, Faculty of Science and Humanities, Shaqra University, Afif, Saudi Arabia \\ ${ }^{4}$ Department of Mathematics, College of Sciences, King Khalid University, Abha 61413, Saudi Arabia \\ ${ }^{5}$ Department of Mathematics, University of the Poonch Rawalakot, Rawalakot 12350, Pakistan
}

Correspondence should be addressed to Taseer Muhammad; taseer_qau@yahoo.com

Received 17 July 2021; Revised 15 December 2021; Accepted 22 December 2021; Published 1 February 2022

Academic Editor: Amin Jajarmi

Copyright (c) 2022 Muzamil Hussain et al. This is an open access article distributed under the Creative Commons Attribution License, which permits unrestricted use, distribution, and reproduction in any medium, provided the original work is properly cited.

\begin{abstract}
Electromagnetic water/CNTs nanofluid flow across a convectively heated moving surface is reported in this communication. Aspect of thermal radiations is considered for heat transport analysis. The concept of nonsimilar boundary layer is executed to simplify the convoluted mathematical expressions. Also, an entropy generation model is considered since its reduction minimizes the loss of available energy, which improves thermal efficiency. The governing model is reduced to a dimensionless system by using an appropriate nonsimilarity transformation. The numerical solution for the velocity and temperature profiles has been obtained by implementing local nonsimilarity via finite difference based Matlab algorithm bvp4c for various quantities of the main emerging parameters. The outcomes are depicted in tabular and graphical formats to analyze impacts of different geometrical, thermophysical, and dynamical factors on temperature, velocity, frictional drag, entropy generation (EG), Nusselt number, and the Bejan number. The temperature profile is seen to rise with Biot number and thermal radiation. Higher radiation parameters and nanoparticle concentrations cause an increase in entropy generation. Horizontal plate with the wedge angle $m=0$ is the optimal geometry for minimizing entropy generation. The increase in the values electric field parameter leads to the rise in the skin friction coefficient. Also, Nusselt number declines when magnetic parameter and Eckert number are increase. The authors discussed the local nonsimilarity approach for simulating the dimensionless nonsimilar structure. To the best of authors' knowledge, no such study has yet been published in the literature. To show the originality of results, the current numerical findings are compared with the published research for some limiting cases and are found to be in excellent alignment. This study could be useful for examining the impacts of nanofluids in a thermal transport analysis.
\end{abstract}

\section{Introduction}

Carbon nanotubes are an allotrope of carbon with tubal nanostructure. Carbon nanotubes have a broad variety of uses, including oil and gas industry, conductive fabrics, atomic force microscope tips, flat-panel displays, radarabsorbing coating, longer-lasting batteries, structural composite materials, antifouling paint, ultracapacitors, and medical instruments and biosensors due to their higher chemical compatibility with biomolecules such as proteins and DNA, as well as purification of tainted drinking water. Compared with other nanostructured compounds, nanotubes have the best thermal conductivity enhancement property. Iijima [1] discovered carbon nanotubes in 1991 by observing the electrical discharge between two carbon electrodes. CNTs are characterized as single wall carbon 
nanotubes and the multiwall carbon nanotubes, all of which depend on concentric layers of rolled graphene lamina. Carbon nanotubes' thermal conductivity is found to be higher than that of pure fluids, and as a result, there is rising preference in SWCNTs and MWCNTs applications in industry, electronics, and medicine [2-4]. Meanwhile, significant work on CNTs boundary layer flow has been documented by [5-7]. Shahsavar et al. [8] examined thermal conductivity of a magnetic hybrid $\left(\mathrm{CNTs}+\mathrm{Fe}_{3} \mathrm{O}_{4}\right)$ nanofluid flow experimentally. Lu et al. [9] discussed the CNTs-suspended nanofluids flow with binary chemical reactions and Cattaneo-Christov heat flux condition. Haq et al. [10] analyzed the velocity slip effects on two-dimensional (2D), steady, incompressible, nanofluid flow containing CNTs over a flat stretching plate. Mosayebidorcheh and Hatami [11] investigated nanofluid (CNTs-water) flow between two parallel rotating disks. Iqbal et al. [12] numerically analyzed the bioconvection, CNTs nanofluid flow across a stretching surface. Lu et al. [13] evaluated numerically the nanofluid flow comprising CNTs with magnetohydrodynamics (MHD) and homogeneous-heterogeneous reactions. Ahmed et al. [14] examined incompressible, 2D, steady boundary layer flow with base fluid (engine oil) in the existence of CNTs with double stratification effects. Upreti et al. [15] assessed the 3D Darcy-Forchheimer water-CNTs (SWCNT and MWCNT) nanofluid flow across 2D stretchable surface with the consequences of Ohmic heating and nonuniform heat source/sink.

Entropy generation (EG) is accounted in technology, heat-related industries, and everyday life events. Thermal energy is converted to mechanical work through entropy, which is the indicator of thermal system disruption. In the form of a campfire and the combustion of solid wood into ash, smoke, and fumes, human everyday life is inextricably linked with entropy. Regarding fluid efficiency in thermal systems, liquids are treated in such a manner that energy waste is minimized. In support of this idea, the concept of EG has gained prominence in the thermal world. Bejan [16] initially investigated entropy production and developed a thermodynamic optimization process. Soomro et al. [17] numerically examined EG for mixed convection CNTs fluid flow over an inclined surface. Ishaq et al. [18] studied nanofluid flow with EG across an unsteady stretching surface. Farooq et al. [19] studied viscous dissipation and transpiration effects on EG in the nanofluid flow across a nonlinear stretched porous disk. Suleman et al. [20] discussed analytically, EG, and thermal radiation effects for nanofluid flow across an exponentially stretching surface. Sheikholeslami et al. [21] used a non-Darcy model to study EG and magnetic nanofluid flow in a porous enclosure. Khan et al. [22] evaluated the EG in nanofluid flow through porous medium comprising microorganisms and nanoparticles. Some important studies on EG have been conducted in [23-29].

Boundary layers theory plays a critical role in an extensive range of engineering and scientific fields. By observing high-temperature processes, such as nuclear power plants, energy storage, and gas turbines, the impact of thermal radiations on boundary-layer flows is crucial. Heat transfer through surface convection across different geometries has obtained a lot of consideration because of its possible uses in a variety of engineering technologies. Many researchers have conducted studies in this region. Ishak et al. [30] studied the convective flow across moving-plate with thermal radiation. Akbar et al. [31] analyzed the incompressible nanofluid flow over a cylinder along with convective boundary condition. Nadeem and Haq [32] explored the magnetohydrodynamic nanoliquids transport across stretching-sheet with the impact of convective-surface. Delouei et al. [33] investigated the boundary layer flow and heat transfer of non-Newtonian fluid over a heated cylinder by employing the direct-forcing IB-TLBM. Oyelakin et al. [34] observed Soret and Dufour effects in Casson nanofluid flow across an unsteady stretched surface with thermal radiation effects. Zaib et al. [35] studied the impacts of mixed convection and thermal radiation on copper-water nanofluid flow over a porous shrinking cylinder with heterogeneous-homogeneous reactions. Jyothi et al. [36] numerically assessed the boundary layer nanofluid flow between two disks employing thermal radiation and convective boundary surface. Reddy et al. [37] examined the influence of Arrhenius activation energy, nonlinear thermal radiations, and binary chemical reaction on MHD boundary layer flow of Eyring-Powell fluid over a stretching surface. Saif et al. [38] reviewed viscous fluid flow across a stretchable curved surface through convective boundary conditions. Raees et al. [39] evaluated mixed convective boundary-layer magnetized second grade nanofluid flow over a vertically stretching sheet. Farooq et al. [40] analyzed nonsimilar boundary-layer flow of Casson nanofluid within non-Darcy porous media.

According to the previously mentioned literature review, nonsimilar EG analysis for an electromagnetic, radiating nanofluids (water-SWCNT/MWCNT) flow across convective heated-wedge, vertical plate, and horizontal-plate has not been published. Thus, the current work aims to address this void in the literature by conducting a systematic review of boundarylayer flow and EG research. The governing equations are numerically solved by utilizing local nonsimilarity method via in-built MATLAB bvp4c. For SWCNT/MWCNT-water nanofluids, the impact of incorporated physical parameters on velocity, temperature, viscous drag, Nusselt number, EG, and Bejan number has been examined.

\section{Mathematical Modeling}

Consider the 2D, incompressible, viscous carbon nanotubesbased flow over a wedge. Water is considered as base fluid, while SWCNTs and MWCNTs are picked as nanoparticles. Free stream and wedge velocities are $U_{e}=U_{\infty} x^{m}$ and $U_{w}=U_{W} x^{m}$, respectively, where $0<m=\beta /(2-\beta)<1, U_{\infty}$ and $U_{w}$ are constant . $\beta=\omega / \pi$ is Hartree pressure gradient parameter; $\omega$ represented a total wedge angle. The case $\beta=0$ relates to a horizontal plate and $\beta=1$ indicates a vertical plate. We presume that $x$ - axis is parallel to the wedge and $y$ - axis in the normal direction of flow. Furthermore, it is anticipated that the wedge's lower surface is heated by heat convection from hot fluid with transfer coefficient $h_{f}$ at a temperature $T_{f}$. The base fluid (water) and the nanoparticles 
(SWCNTs and MWCNTs) are likewise considered to be in equilibrium. Figure 1 reveals the schematic sketch of the problem.
Governing equations for the problems under consideration [41] are as follows:

$$
\begin{aligned}
\frac{\partial u}{\partial x}+\frac{\partial v}{\partial y}= & 0 \\
u \frac{\partial u}{\partial x}+v \frac{\partial u}{\partial y}= & -\frac{1}{\rho_{n f}} \frac{\partial p}{\partial x}+\frac{\mu_{n f}}{\rho_{n f}} \frac{\partial^{2} u}{\partial y^{2}}+\frac{\sigma_{n f}}{\rho_{n f}}\left(E_{0} B_{0}-B_{0}^{2} u\right) \\
u \frac{\partial T}{\partial x}+v \frac{\partial T}{\partial y}= & \alpha \frac{\partial^{2} T}{\partial y^{2}}+\frac{\mu_{n f}}{\left(\rho c_{p}\right)_{n f}}\left(\frac{\partial u}{\partial y}\right)^{2} \\
& -\frac{1}{\left(\rho c_{p}\right)_{n f}} \frac{\partial q_{r}}{\partial y}+\frac{\sigma_{n f}}{\left(\rho c_{p}\right)_{n f}}\left(B_{0}^{2} u^{2}-E_{0}^{2}\right)
\end{aligned}
$$

where $u$ and $v$ are the $x$ - and $y$-directions velocity components, respectively. $T$ is the nanofluid temperature and $\mu_{n f}, \rho_{n f}, \alpha_{n f}, k_{n f}$, and $\left(\rho c_{p}\right)_{n f}$ are the nanofluid's dynamic viscosity, density, thermal diffusivity, effective thermal conductivity, and heat capacitance.

In an inviscid flow, velocity at edge of the boundary layer and mainstream velocity $\left(U_{e}\right)$ are identical, and pressure is treated as constant. From (2), we have

$$
U_{e}(x) \frac{\partial U_{e}(x)}{\partial x}=-\frac{1}{\rho_{n f}} \frac{\partial p}{\partial x} .
$$

Employing equation (4) into equation (2), we get

$$
\begin{aligned}
u \frac{\partial u}{\partial x}+v \frac{\partial u}{\partial y}= & U_{e}(x) \frac{\partial U_{e}(x)}{\partial x}+\frac{\mu_{n f}}{\rho_{n f}} \frac{\partial^{2} u}{\partial y^{2}} \\
& +\frac{\sigma_{n f}}{\rho_{n f}}\left(E_{0} B_{0}-B_{0}^{2} u\right) .
\end{aligned}
$$

Rosseland approximation is considered [36]:

$$
q_{r}=-\frac{4 \sigma^{*}}{3 k^{*}} \frac{\partial T^{4}}{\partial y}
$$

where $\left(k^{*}\left(m^{-1}\right)\right)$ and $\left(\sigma^{*}=5.67 \times 10^{-9}\left(\mathrm{~W} / \mathrm{m}^{2} \mathrm{k}^{2}\right)\right)$ represents Rosseland absorption coefficient and Stephan-Boltzmann constant. Consider that the temperature variations in the flow are minimal, so $T^{4}$ can be written as

$$
T^{4} \approx 4 T_{\infty}^{3} T-3 T_{\infty}^{4}
$$

Employing equations (6)-(7) into equation (3), we get

$$
u \frac{\partial T}{\partial x}+v \frac{\partial T}{\partial y}=\left[\alpha_{n f}+\frac{16 \sigma^{*} T_{\infty}^{3}}{3 k^{*}\left(\rho c_{p}\right)_{n f}}\right] \frac{\partial^{2} T}{\partial y^{2}}+\frac{\mu_{n f}}{\left(\rho c_{p}\right)_{n f}}\left(\frac{\partial u}{\partial y}\right)^{2}+\frac{\sigma_{n f}}{\left(\rho c_{p}\right)_{n f}}\left(B_{0}^{2} u^{2}-E_{0}^{2}\right) .
$$

The linked boundary conditions (BCs) are

Dimensionless transformations [41] are as follows:

$$
\begin{aligned}
& u=U_{w}(x)=U_{w} x^{m}, v=0,-k_{n f} \frac{\partial T}{\partial y}=h_{f}\left(T_{f}-T\right), \text { at }(y=0), \\
& u=U_{e}(x)=U_{\infty} x^{m}, T=T_{\infty} \text { as }(y \longrightarrow \infty) .
\end{aligned}
$$

$$
\begin{aligned}
& \eta=y \sqrt{\frac{(m+1) U_{e}(x)}{2 v_{b f} x}}, \xi=\frac{x}{l}, u=U_{e} \frac{\partial f}{\partial \eta} \\
& v=\sqrt{\frac{2 v_{b f} U_{e}(x)}{(m+1) x}}\left\{\left(\frac{m+1}{2}\right) f(\xi, \eta)+\xi \frac{\partial f}{\partial \xi}+\left(\frac{m-1}{2}\right) \eta \frac{\partial f}{\partial \eta}\right\}, \theta(\xi, \eta)=\frac{T-T_{\infty}}{T_{f}-T_{\infty}} .
\end{aligned}
$$




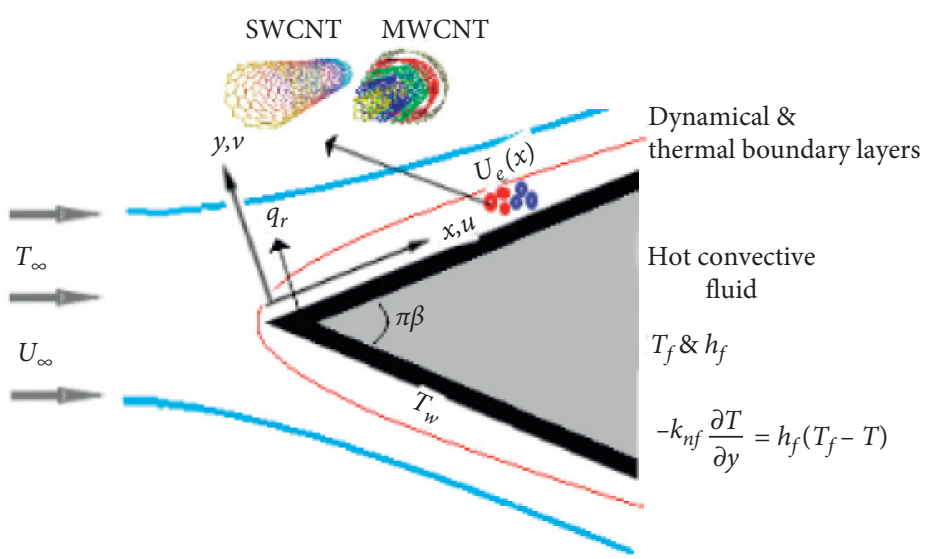

(a)

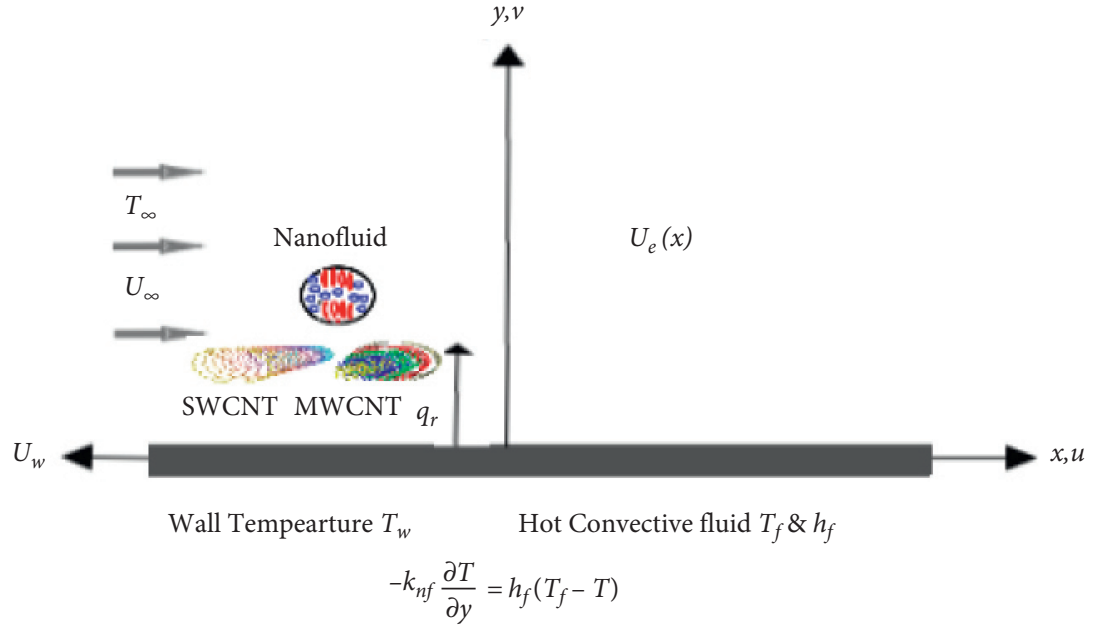

(b)

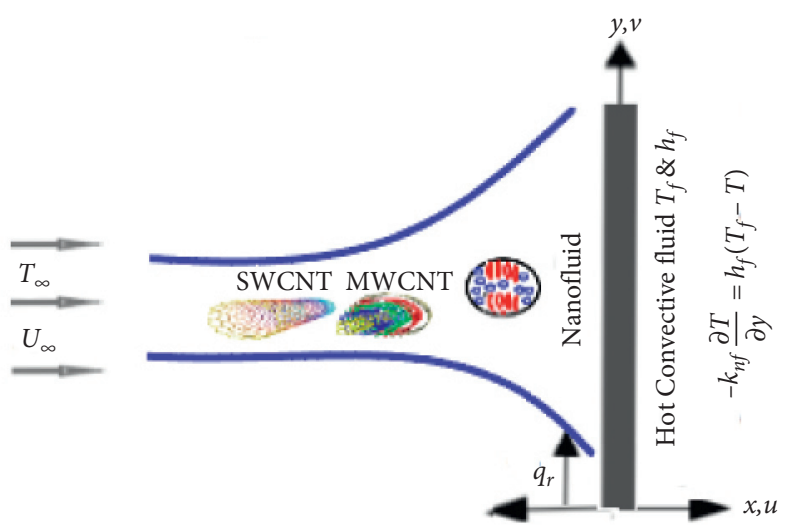

(c)

Figure 1: Physical view of the flow problem. 
Equation (1) is verified automatically, while (2)-(9) become

$$
\begin{aligned}
& m\left(\frac{\partial f}{\partial \eta}\right)^{2}-\frac{(m+1)}{2} f \frac{\partial^{2} f}{\partial \eta^{2}}-m-\frac{(m+1)}{2 A 1 A 2} \frac{\partial^{3} f}{\partial \eta^{3}}-\frac{A 3}{A 2} \xi^{(1-m)} \\
& M\left(E 1 \xi^{-m}-\frac{\partial f}{\partial \eta}\right)=-\xi\left(\frac{\partial^{2} f}{\partial \xi \partial \eta} \frac{\partial f}{\partial \eta}-\frac{\partial f}{\partial \xi} \frac{\partial^{2} f}{\partial \eta^{2}}\right) \\
& \frac{(m+1)}{2 P r A 4}\left(A 5+\frac{4}{3} N r\right) \frac{\partial^{2} \theta}{\partial \eta^{2}}+\frac{1}{A 1 A 4} E c \xi^{2 m}(m+1)\left(\frac{\partial^{2} f}{\partial \eta^{2}}\right)^{2} \\
& +\frac{A 3}{A 4} \xi^{(1+m)} M E c\left(\left(\frac{\partial f}{\partial \eta}\right)^{2}-\xi^{-2 m} E_{1}^{2}\right)+\frac{(m+1)}{2} f \frac{\partial \theta}{\partial \eta}=\xi\left(\frac{\partial f}{\partial \eta} \frac{\partial \theta}{\partial \xi}-\frac{\partial f}{\partial \xi} \frac{\partial \theta}{\partial \eta}\right)
\end{aligned}
$$

With boundary conditions, the following is obtained:

$$
\begin{aligned}
& \frac{\partial f}{\partial \eta}(\xi, 0)=\lambda, \frac{(m+1)}{2} f(\xi, 0)+\xi \frac{\partial f}{\partial \xi}(\xi, 0)=0, \\
& \frac{\partial \theta}{\partial \eta}(\xi, 0)=-\frac{B i}{A 5}(1-\theta(\xi, 0)) \xi^{-m} \sqrt{\frac{2}{(m+1)}}, \\
& \frac{\partial f}{\partial \eta}(\xi, \infty)=1, \theta(\xi, \infty)=0 .
\end{aligned}
$$

Here, $m$ denotes the dimensionless wedge angle; $\lambda$ represents the velocity ratio parameter; $\lambda=0$ depicts static wedge; $\lambda>0$ and $\lambda<0$ show moving-wedge situation in the same and opposite direction to free stream direction, respectively. $\mathrm{Nr}$ expresses the radiation parameter, $\mathrm{Re}$ indicates the Reynolds-number, $B i$ is the Biot number (surface convection parameter), $E c$ and $\operatorname{Pr}$ represent Eckert and Prandtl numbers, respectively, $E_{1}$ is the electric field parameter, and $M$ is the Hartmann number defined as

$$
\begin{gathered}
\lambda=\frac{U_{w}}{U_{e}}, N r=\frac{4 \sigma^{*} T_{\infty}^{3}}{k^{*}(k)_{b f}}, \operatorname{Pr}=\frac{v_{b f}}{\alpha_{b f}}, E c=\frac{\left(U_{\infty} l^{m}\right)^{2}}{c_{p} \Delta T}, B i=\frac{h l}{k_{b f}}(\mathrm{Re})^{-1 / 2}, \\
\operatorname{Re}=\frac{U_{\infty} l^{m+1}}{v_{b f}}, E_{1}=\frac{E_{0}}{B_{0} U_{\infty} l^{m}}, M=\frac{\sigma_{b f} B_{0}^{2}}{\rho_{b f} U_{\infty} l^{m-1}}, A 1=(1-\phi)^{2.5}, A 2=(1-\phi) \\
+\phi \frac{\rho_{C N T}}{\rho_{b f}}, A 3=\frac{\sigma_{n f}}{\sigma_{b f}}, A 4=(1-\phi)+\phi \frac{\left(\rho c_{p}\right)_{C N T}}{\left(\rho c_{p}\right)_{b f}}, A 5=\frac{k_{n f}}{k_{b f}},
\end{gathered}
$$

where $\varphi$ is the nanofluid volume fraction, and $\rho_{b f}, k_{b f}$, and $\sigma_{b f}$ are the density, thermal conductivity, and electrical conductivity of the base fluid (see Table 1).
2.1. First Truncation System. At the first truncation level, the terms accompanied by $\xi \partial(.) / \partial \xi$ are considered approximately very small. This is particularly true when $\xi \ll 1$. Therefore, (11)-(13) become

$$
\begin{aligned}
& m f^{\prime 2}-\frac{(m+1)}{2} f f^{\prime \prime}-m-\frac{(m+1)}{2 A 1 A 2} f^{\prime \prime \prime}-\frac{A 3}{A 2} \xi^{(1-m)} M\left(E 1 \xi^{-m}-f_{\prime}\right)=0, \\
& \frac{(m+1)}{2 \operatorname{Pr} A 4}\left(A 5+\frac{4}{3} N r\right) \theta \prime \prime+\frac{1}{A 1 A 4} E c \xi^{2 m}(m+1) f^{\prime \prime 2} \\
& +\frac{(m+1)}{2} f \theta \prime+\frac{A 3}{A 4} \xi^{(1+m)} M E c\left(f \prime^{2}-\xi^{-2 m} E_{1}^{2}\right)=0 .
\end{aligned}
$$


TABLE 1: The model for thermophysical properties [15].

\begin{tabular}{lcc}
\hline Property & Symbol & Defined \\
\hline Viscosity & $\mu_{n f}$ & $\mu_{n f}=\mu_{b f} /(1-\phi)^{2,5}$ \\
Density & $\rho_{n f}$ & $\rho_{n f}=(1-\phi) \rho_{b f}+\phi \rho_{C N T}$ \\
Heat capacitance & $\left(\rho C_{p}\right)_{n f}$ & $\left(\rho C_{p}\right)_{n f}=(1-\phi)\left(\rho C_{p}\right)_{b f}+\phi\left(\rho C_{p}\right)_{C N T}$ \\
Electric conductivity & $\sigma_{n f}$ & $\sigma_{n f}=\left\{1+\frac{3\left(\sigma_{C N T} / \sigma_{b f}-1\right) \phi}{\left(\sigma_{C N T} / \sigma_{b f}+2\right)-\left(\sigma_{C N T} / \sigma_{b f}-1\right) \phi}\right\} \sigma_{b f}$ \\
Thermal conductivity & & $\frac{k_{n f}}{k_{b f}}=\frac{1-\phi+2 \phi\left(k_{C N T} /\left(k_{C N T}-k_{b f}\right)\right) \ln \left(\left(k_{C N T}+k_{b f}\right) / 2 k_{b f}\right)}{1-\phi+2 \phi\left(k_{b f} /\left(k_{C N T}-k_{b f}\right)\right) \ln \left(\left(k_{C N T}+k_{b f}\right) / 2 k_{b f}\right)}$ \\
\hline
\end{tabular}

Boundary conditions are as follows:

$$
\begin{aligned}
f^{\prime}(\xi, 0) & =\lambda, \frac{(m+1)}{2} f(\xi, 0)=0, \\
\theta^{\prime}(\xi, 0) & =-\frac{B i}{A 5}(1-\theta(\xi, 0)) \xi^{-m} \sqrt{\frac{2}{(m+1)}}, \\
f^{\prime}(\xi, \infty) & =1, \theta(\xi, \infty)=0 .
\end{aligned}
$$

2.2. Second Truncation System. For the second truncation system, we assumed the followings:

$$
\frac{\partial f}{\partial \xi}=g, \frac{\partial \theta}{\partial \xi}=h, \text { and } \frac{\partial g}{\partial \xi}=\frac{\partial h}{\partial \xi}=0 .
$$

Therefore, equations (11)-(13) take the following form:

$$
\begin{aligned}
& g^{\prime \prime \prime}=\frac{2 A 1 A 2}{m+1}\left(\begin{array}{c}
2 m g^{\prime} f^{\prime}-\frac{m+1}{2}\left(g f^{\prime \prime}+f g^{\prime \prime}\right)+g^{\prime} f^{\prime}-f^{\prime \prime} g+\xi\left(g^{\prime 2}-g^{\prime \prime} g\right) \\
-\frac{A 3}{A 2} M \xi^{-m}\left((1-m)\left(E 1 \xi^{-m}-f^{\prime}\right)+\xi\left(-m \xi^{-m-1} E 1-g^{\prime}\right)\right)
\end{array}\right), \\
& h^{\prime \prime}=\frac{2 \operatorname{Pr}}{(m+1)}\left(\begin{array}{c}
-\left(\frac{m+3}{2}\right) g \theta^{\prime}+h f^{\prime}-\left(\frac{m+1}{2}\right) f h^{\prime}+\xi\left(h g^{\prime}-g h^{\prime}\right) \\
-2 E c \xi^{2 m}\left(\frac{m+1}{A 1 A 4}\right)\left(m \xi^{-1} f^{\prime \prime^{2}}+f^{\prime \prime} g^{\prime \prime}\right)-\frac{A 3}{A 2} M E c \xi^{m}\left((1+m)\left(f^{\prime^{2}}-\xi^{-2 m} E 1^{2}\right)+2 \xi\left(g^{\prime} f^{\prime}+m \xi^{-2 m-1} E 1^{2}\right)\right)
\end{array}\right) .
\end{aligned}
$$

Boundary conditions are as follows:

$$
\begin{aligned}
g^{\prime}(0, \xi) & =0, g(0, \xi)=0, h^{\prime}(0, \xi) \\
& =-\frac{\xi^{-m}}{A 5} \sqrt{\frac{2}{m+1}} B i\left(-m \xi^{-m}(1-\theta)-h\right), g^{\prime}(\infty, \xi) \\
& =0, h(\infty, \xi)=0 .
\end{aligned}
$$

Frictional drag on the surface (local skin friction coefficient $C_{f}$ ) and rate of heat transfer (local Nusselt number $N u_{x}$ ) are defined as

$$
C_{f}=\frac{\tau_{w}}{\rho_{n f} U_{e}^{2}}, N u_{x}=\frac{x q_{w}}{k_{b f}\left(T_{f}-T_{\infty}\right)},
$$

where $\quad \tau_{w}=\mu_{n f}(\partial u / \partial y)_{y=0} \quad$ and $\quad q_{w}=-\left[k_{n f}+16 \sigma^{*} T_{\infty}^{3}\right.$ $\left./ 3 k^{*}\right](\partial T / \partial y)_{y=0}$ are the shearing stress and heat flux at the surface of the wall, respectively. Putting in (15), we get

$$
\begin{aligned}
C_{f} R_{e}^{1 / 2} & =\frac{1}{A 1 A 2} \sqrt{\frac{(m+1)}{2}} \xi^{-(m+1) / 2} f^{\prime \prime}(0), N u R_{e}^{-1 / 2} \\
& =\left(A 5+\frac{4 N r}{3}\right) \sqrt{\frac{(m+1)}{2}} \xi^{(m+1) / 2} \theta^{\prime}(0) .
\end{aligned}
$$

\section{Entropy Generation Analysis}

Entropy generation (EG) per unit volume for the problem under consideration is [15]

$$
S_{G}=\underbrace{\frac{k_{n f}}{T_{\infty}^{2}}\left[1+\frac{16 \sigma^{*} T_{\infty}^{3}}{3 k^{*} k_{b f}}\right]\left(\frac{\partial T}{\partial y}\right)^{2}}_{\text {Heat Transfer irreversibility }}+\underbrace{\frac{\mu_{n f}}{T_{\infty}}\left(\frac{\partial u}{\partial y}\right)^{2}}_{\text {viscous dissipation irreversibility }}+\underbrace{\frac{\sigma_{n f}}{T_{\infty}}\left(B_{0}^{2} u^{2}-E_{0}^{2}\right)}_{\text {Joule heating irreversibility }}
$$


The dimensionless entropy generation number $\left(N_{G}\right)$ is defined as

$$
\begin{aligned}
N_{G}= & \frac{S_{G}}{S_{G O}}=\operatorname{Re}\left(\frac{m+1}{2}\right)\left[\xi^{(m-1)} A 5\left(1+\frac{4}{3} N r\right)\left(\frac{\partial \theta}{\partial \eta}\right)^{2}+\xi^{(3 m-1)}\left(\frac{1}{A 1} \frac{E c \operatorname{Pr}}{\Omega}\right)\left(\frac{\partial^{2} f}{\partial \eta^{2}}\right)^{2}\right. \\
& \left.+A 3 \xi^{2 m}\left(\frac{2}{m+1}\right) M E c \operatorname{Pr}\left(\left(\frac{\partial f}{\partial \eta}\right)^{2}-\xi^{-2 m} E 1^{2}\right)\right],
\end{aligned}
$$

where $\Omega^{-1}=T_{\infty} / \Delta T$ represents the dimensionless temperature difference and $S_{G O}=k_{b f} \Delta T^{2} / T_{\infty}^{2} l^{2}$ denotes the characteristic entropy generation rate.
Dimensionless form of Bejan number $(B e)$ for the considered problem can be stated as

$$
\begin{aligned}
& B e=\frac{\text { entropy generation due to heat transfer }}{\text { total entropy generation }}, \\
& B e=\frac{\xi^{(m-1)} A 5(1+4 / 3 N r)(\partial \theta / \partial \eta)^{2}}{\xi^{(m-1)} A 5(1+4 / 3 N r)(\partial \theta / \partial \eta)^{2}+\xi^{(3 m-1)}(1 / A 1 E c \operatorname{Pr} / \Omega)\left(\partial^{2} f / \partial \eta^{2}\right)^{2}+A 3 \xi^{2 m}(2 / m+1) M E c \operatorname{Pr}\left((\partial f / \partial \eta)^{2}-\xi^{-2 m} E 1^{2}\right)}
\end{aligned}
$$

\section{Numerical Solution Procedure}

In this section, we will discuss the numerical solution procedure for the dimensionless equations (15) to (21) using Matlab algorithm bvp4c. The ordinary differential equations (ODEs) solver technique employs the Lobatto IIIA formula, which necessitated the initial guesses to justify the boundary conditions. Hereafter, we employ FDM, which updates the initial predictions for the subsequent iterations. We accomplish the process with the following assumptions:

$$
\begin{aligned}
& f=\left.\right|_{1}, f^{\prime}=\left.\right|_{2}, g^{\prime \prime}=\left.\right|_{3}, \theta=\left.\right|_{4}, \theta^{\prime}=\left.\right|_{5}, \\
& g=\left.\right|_{6}, g^{\prime}=\left.\right|_{7}, g^{\prime \prime}=\left.\right|_{8}, h=\left.\right|_{9}, h^{\prime}=\left.\right|_{10} .
\end{aligned}
$$

\section{Results and Discussion}

Electromagnetic, radiating water-CNTs nanofluid flow over convective heated-wedge and vertical/horizontal surface under impact of entropy optimization has been investigated by employing the method of local nonsimilarity [42] up to the second truncation level via Matlab command bvp $4 \mathrm{c}$ numerically. According to the total wedge angle $\omega$, three cases are examined for boundary layer flow. The flow around a flat plate is represented by $\beta=0$ or $(m=0), \beta=1$ or $(m=1)$, which shows the flow across a vertical plate, and for the wedge flow, we choose $\beta=1 / 2$ or $(m=1 / 3)$. Values of $\beta$ approximately correlate to the wedge angles $\omega=\pi \beta=0^{\circ}, 90^{\circ}$, and $180^{\circ}$. The Prandtl number (Pr) is fixed as 6.2, and nanoparticles volume fraction $(\phi)$ is examined in $0 \leq \phi \leq 0.2$ range. The physical importance of various flow fields has been demonstrated for several relevant parameters. We set the parameters $\phi=0.1, M=1.5, E 1=0.1, N r=0.5, E c$ $=0.1, \lambda=0.5$, and $B i=0.4$, unless otherwise specified in specific figures/tables. Table 2 implies the thermophysical properties of water and both CNTs. To assess the authenticity of the present solutions, Table 3 reports a comparison examination of $f^{\prime \prime}(0)$ for several values of with earlier published results, which validates our method. Table 4 depicts the skin friction coefficient's $C_{f} R_{e}^{1 / 2}$ behavior in response to various estimations of $\phi, m, \lambda, M$, and $E_{1}$ for both MWCNT $+\mathrm{H}_{2} \mathrm{O}$ and SWCNT $+\mathrm{H}_{2} \mathrm{O}$. Viscous drag is reduced as $\lambda$ and $M$ are increased. Further, rise in $\phi, m$, and $E_{1}$ upgrades the viscous drag for both MWCNT/SWCNT-water nanofluid. The numerical value of the Nusselt number is shown in Table 5 for both SWCNTs and MWCNTs. Nusselt number $(\mathrm{Nu})$ decreases for greater values of magnetic parameter and Eckert number, while it rises for increasing the values of dimension less electric field, surface convection, velocity ratio parameter, solid volume fraction, and radiation parameters.

Velocity $\left(f^{\prime}(\eta)\right)$ for several values of the $(\phi)$ are shown in Figure 2. It shows that when $\phi$ rises, $f^{\prime}(\eta)$ falls for both conducting SWCNT/MWCNT-water nanofluids flow by wedge $(m=1 / 3)$ and a horizontal $(m=0) /$ vertical plate $(m=1)$. By raising $\phi$, the fluid's viscosity enhances and so resists the flow under the impact of shear stress. As a result, fluid velocity diminishes. The significance of magnetic parameter $(M)$ on the velocity field for all cases are outlined in Figure 3. For greater estimations of $M$, velocity decreases. Increase in $M$ indicates a rise in resistive power (Lorentz force), and so, the velocity of the liquid decreases. Figure 4 indicates that the rise in $E_{1}$ upgrades velocity field irrespective of $m=0,1 / 3,1$. This is because an increase in $E_{1}$ adds to a 
TABLE 2: Thermophysical properties of SWCNTs, MWCNTs, and water [43].

\begin{tabular}{lcccc}
\hline Materials & $c_{p}(\mathrm{~J} / \mathrm{kgK})$ & $\rho\left(\mathrm{kg} / \mathrm{m}^{3}\right)$ & $k(\mathrm{~W} / \mathrm{mK})$ & $\sigma(\Omega m)^{-1}$ \\
\hline SWCNTs & 425 & 2600 & 6600 & $10^{6}-10^{7}$ \\
MWCNTs & 796 & 1600 & 3000 & $1.9 \times 10^{-4}$ \\
Pure water & 4179 & 997.1 & 0.613 & 0.05 \\
\hline
\end{tabular}

TABLE 3: The comparison of $f^{\prime \prime}(0)$ for varying $m$ when $\lambda=\phi=M=E 1=0$.

\begin{tabular}{lcccc}
\hline$m$ & Yih [44] & Yacob et al. [45] & Berrehal et al. [41] & Present study \\
\hline 0 & 0.469600 & 0.469599 & 0.46959999 & 0.4901403164 \\
$1 / 11$ & 0.654979 & 0.654994 & 0.65499369 & 0.6622684297 \\
0.2 & 0.802125 & 0.802126 & 0.80212559 & 0.8052247372 \\
$1 / 3$ & 0.927653 & 0.927680 & 0.92768004 & 0.9291508721 \\
0.5 & - & 1.038903 & 1.03890348 & 1.0396547284 \\
1 & 1.232588 & 1.232588 & 1.23258766 & 1.2328155608 \\
\hline
\end{tabular}

TABLE 4: Skin friction coefficient $C_{f} R_{e}^{1 / 2}$ for different parameters.

\begin{tabular}{|c|c|c|c|c|c|c|}
\hline \multirow{2}{*}{$\phi$} & \multirow{2}{*}{$m$} & \multirow{2}{*}{$\lambda$} & \multirow{2}{*}{$M$} & \multirow{2}{*}{$E_{1}$} & \multicolumn{2}{|c|}{$1 / A 1 A 2 \sqrt{(m+1) / 2} \xi^{-(m+1) / 2} f^{\prime \prime}(0)$} \\
\hline & & & & & $\mathrm{SWCNT}+\mathrm{H}_{2} \mathrm{O}$ & $\mathrm{MWCNT}+\mathrm{H}_{2} \mathrm{O}$ \\
\hline 0.0 & 0.5 & 0.5 & 0.1 & 0.7 & 0.5210884693 & 0.5210884693 \\
\hline 0.1 & & & & & 0.5513761718 & 0.5797717855 \\
\hline 0.2 & & & & & 0.5989160976 & 0.6565461699 \\
\hline \multirow[t]{13}{*}{0.1} & 0.0 & & & & 0.2381317407 & 0.2543772839 \\
\hline & 0.5 & & & & 0.5513761718 & 0.5797717855 \\
\hline & 1.0 & & & & 0.7489760758 & 0.7857562834 \\
\hline & 0.5 & -0.5 & & & 1.2001180242 & 1.2346030460 \\
\hline & & 0.5 & & & 0.5513761718 & 0.5797717855 \\
\hline & & 1.0 & & & -0.0335533455 & -0.0246257247 \\
\hline & & 1.5 & & & -0.7490392975 & -0.7669070898 \\
\hline & & 0.5 & 0.1 & & 0.5513761718 & 0.5797717855 \\
\hline & & & 0.2 & & 0.5446412999 & 0.5746013141 \\
\hline & & & 0.3 & & 0.5392774702 & 0.5701587146 \\
\hline & & & 0.1 & 0.5 & 0.5274159969 & 0.5621837556 \\
\hline & & & & 0.6 & 0.5394093940 & 0.5709844630 \\
\hline & & & & 0.7 & 0.5513761718 & 0.5797717855 \\
\hline
\end{tabular}

TABLE 5: Nusselt number $N u_{x} R_{e}^{-1 / 2}$ for different parameters.

\begin{tabular}{|c|c|c|c|c|c|c|c|c|c|}
\hline \multirow{2}{*}{$\phi$} & \multirow{2}{*}{$m$} & \multirow{2}{*}{$\lambda$} & \multirow{2}{*}{$M$} & \multirow{2}{*}{$E_{1}$} & \multirow{2}{*}{$\mathrm{Nr}$} & \multirow{2}{*}{$E c$} & \multirow{2}{*}{$B i$} & \multicolumn{2}{|c|}{$(A 5+4 N r / 3) \sqrt{(m+1) / 2} \xi^{(m+1) / 2} \theta \prime(0)$} \\
\hline & & & & & & & & $\mathrm{SWCNT}+\mathrm{H}_{2} \mathrm{O}$ & $\mathrm{MWCNT}+\mathrm{H}_{2} \mathrm{O}$ \\
\hline 0.0 & 0.5 & 0.5 & 0.1 & 0.7 & 1.0 & 0.1 & 0.1 & 0.5828954946 & 0.5828954946 \\
\hline 0.1 & & & & & & & & 0.6714426295 & 0.6676208212 \\
\hline 0.2 & & & & & & & & 0.7191203712 & 0.7159706891 \\
\hline \multirow[t]{15}{*}{0.1} & 0.0 & & & & & & & 0.6364652976 & 0.6306984674 \\
\hline & 0.3 & & & & & & & 0.6591926637 & 0.6548984620 \\
\hline & 1.0 & & & & & & & 0.6959009226 & 0.6927497425 \\
\hline & 0.5 & -0.5 & & & & & & 0.0587727263 & 0.0797575148 \\
\hline & & 0.0 & & & & & & 0.4611064766 & 0.4695032017 \\
\hline & & 0.5 & & & & & & 0.6676208212 & 0.6714426295 \\
\hline & & 0.5 & 0.1 & & & & & 0.6714426295 & 0.6676208212 \\
\hline & & & 0.2 & & & & & 0.6676597316 & 0.6652136735 \\
\hline & & & 0.3 & & & & & 0.6643572299 & 0.6630236826 \\
\hline & & & 0.1 & 0.5 & & & & 0.6652479312 & 0.6635737207 \\
\hline & & & & 0.6 & & & & 0.6680242796 & 0.6653834823 \\
\hline & & & & 0.7 & & & & 0.6714426295 & 0.6676208212 \\
\hline & & & & 0.7 & 0.1 & & & 0.6714426295 & 0.6676208212 \\
\hline & & & & & 0.5 & & & 0.7819621019 & 0.7839066887 \\
\hline & & & & & 1.0 & & & 0.9158431475 & 0.9243077169 \\
\hline
\end{tabular}


TABle 5: Continued.

\begin{tabular}{|c|c|c|c|c|c|c|c|c|c|}
\hline \multirow{2}{*}{$\phi$} & \multirow{2}{*}{$m$} & \multirow{2}{*}{$\lambda$} & \multirow{2}{*}{$M$} & \multirow{2}{*}{$E_{1}$} & \multirow{2}{*}{$\mathrm{Nr}$} & \multirow{2}{*}{$E c$} & \multirow{2}{*}{$B i$} & \multicolumn{2}{|c|}{$(A 5+4 N r / 3) \sqrt{(m+1) / 2} \xi^{(m+1) / 2} \theta \prime(0)$} \\
\hline & & & & & & & & $\mathrm{SWCNT}+\mathrm{H}_{2} \mathrm{O}$ & $\mathrm{MWCNT}+\mathrm{H}_{2} \mathrm{O}$ \\
\hline & & & & & 0.1 & 0.1 & & 0.6714426295 & 0.6676208212 \\
\hline & & & & & & 0.2 & & 0.6225801744 & 0.6214259544 \\
\hline & & & & & & 0.3 & & 0.5737177194 & 0.5752310878 \\
\hline & & & & & & & 1 & 0.6714426295 & 0.6676208212 \\
\hline & & & & & & & 10 & 1.7562062035 & 1.7122962566 \\
\hline & & & & & & & 20 & 1.9293753005 & 1.8753214528 \\
\hline
\end{tabular}

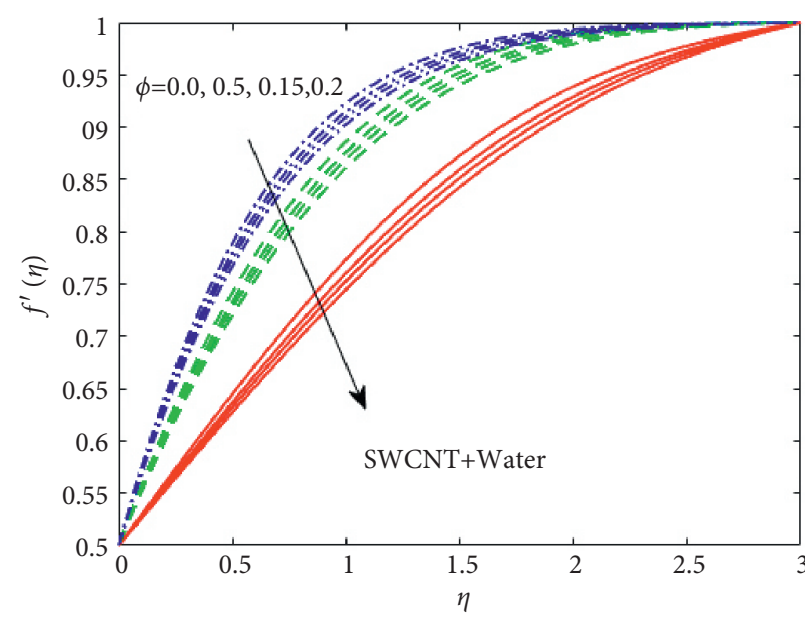

$$
\begin{aligned}
-\mathrm{m} & =0.0 \\
--\mathrm{m} & =1 / 3 \\
---\mathrm{m} & =1.0
\end{aligned}
$$

(a)

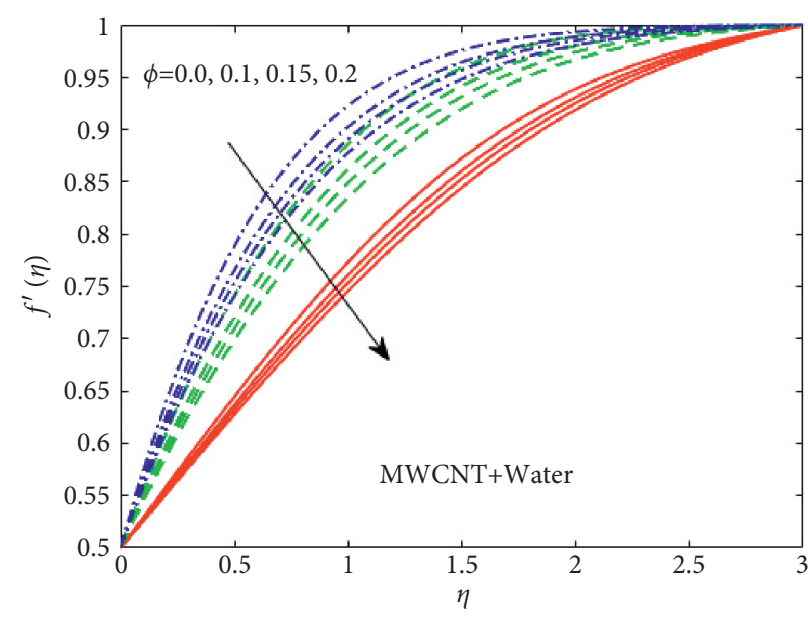

$$
\begin{aligned}
-\mathrm{m} & =0.0 \\
--\mathrm{m} & =1 / 3 \\
--\mathrm{m} & =1.0
\end{aligned}
$$

(b)

Figure 2: $\phi$ influence on $f^{\prime}(\eta)$ for (a) SWCNT + water (b) MWCNT + water.

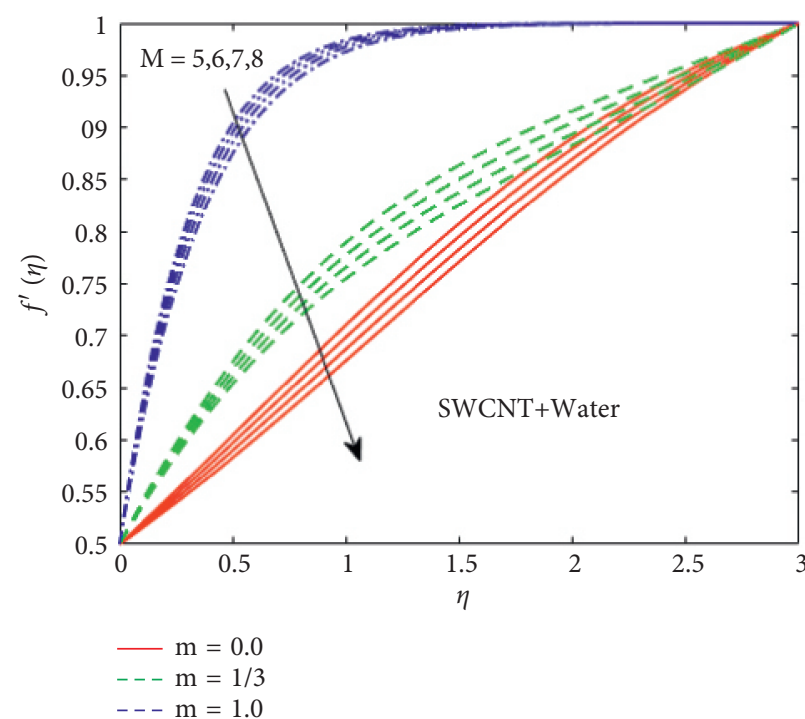

(a)

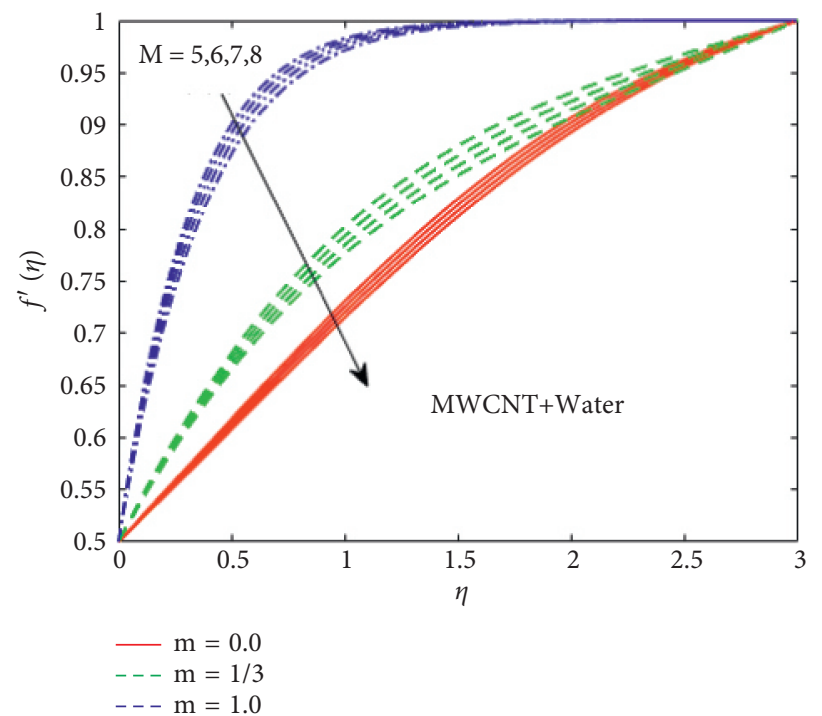

(b)

FIgURE 3: $M$ influence on $f^{\prime}(\eta)$ for (a) SWCNT + water (b) MWCNT + water. 


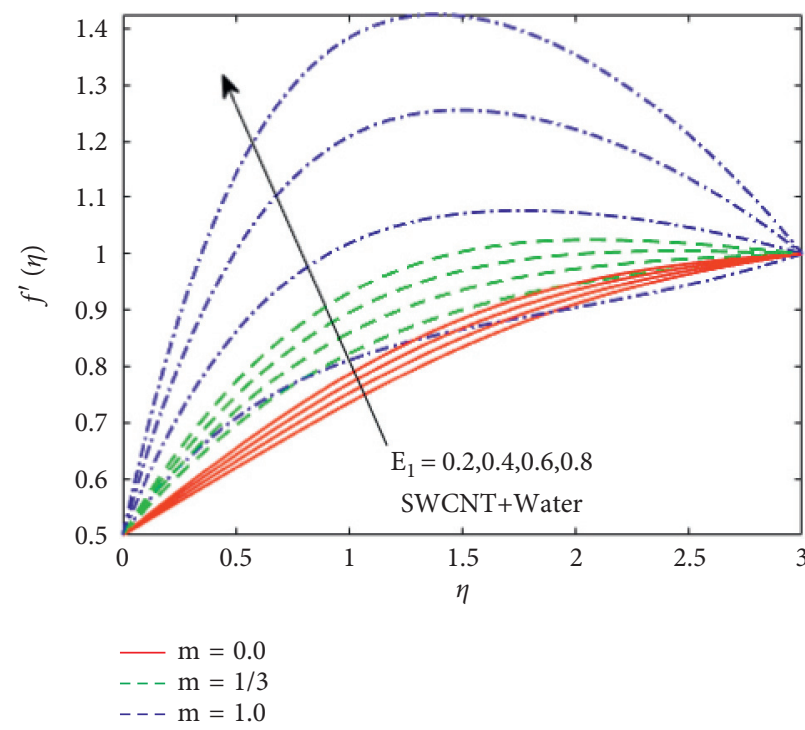

(a)

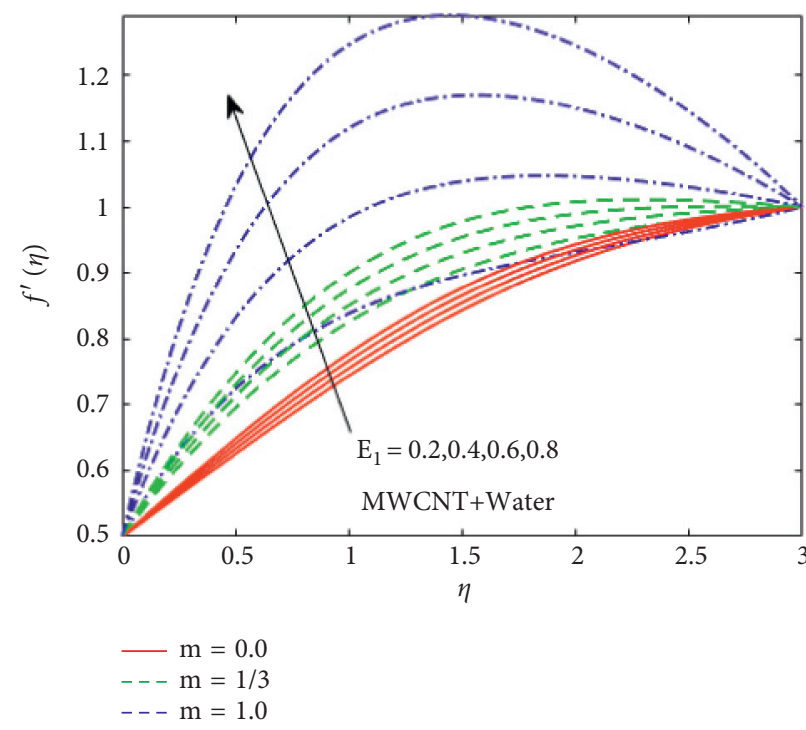

(b)

FIgURE 4: $E_{1}$ influence on $f^{\prime}(\eta)$ for (a) SWCNT + water (b) MWCNT + water.

stronger electric field, which accelerates the flow of CNTs nanofluids. Figure 5 demonstrates the variance in velocity for growing velocity ratio parameter $(\lambda)$. It shows that velocity of nanofluids within the boundary layer rises with $\lambda$.

Influences of nanoparticles fraction $(\phi)$ on $(\theta(\eta))$ are seen in Figure 6. Clearly, graphs show that increasing the nanoparticles concentration raises the temperature inside the boundary layer for all considered cases. This is because as $\phi$ increases, the thermal conductivity of the nanofluid rises, and as a result, the temperature of the fluid grows. Figure 7 outlines the significance of the temperature by considering an estimation of the radiation parameter $(\mathrm{Nr})$. Clearly, increasing the radiation estimator value improves the temperature distribution and corresponding boundary layer thickness. It is evident that thermal radiation causes an upsurge in surface heat flux. Consequently, the temperature rises within the boundary layer region. Figure 8 portrays the effects of Hartmann $(M)$ on temperature profile $\theta(\eta)$. It is clear that as the value of $M$ increases, so does the temperature. The Lorentz force obstructs fluid flow, whereas rise in the Hartmann number increases the Lorentz force, resulting in a portion of energy being transferred as heat. Temperature profile rises as a result. An analysis of Eckert number $(E c)$ on $\theta(\eta)$ is portrayed in Figure 9. It is worth noting that when $E c$ rises, $\theta(\eta)$ increases for both SWCNT and MWCNT. Increase in the Ec results in higher drag forces between the fluid materials. Consequently, more heat is generated, causing the temperature distribution to increase. The behaviors of $\theta(\eta)$ in response to different $E_{1}$ for electromagnetic flow of CNTs nanofluids transport by convective heated-horizontal/vertical plates and wedge surface are drawn in Figure 10. In this case, the temperature distribution for both nanofluids declines as $E_{1}$ increases. The variations of $\theta(\eta)$ with the strength of local Biot number $(B i)$ is seen in Figure 11. Thermal boundary layer thickness was shown to rise slightly with an escalation in the $B i$ for all the considered cases. Biot number is defined physically as the relationship between conduction and convection at the surface. As predicted, stronger convection results in higher surface temperatures. Physically, the inclination in Bi generates significant heat transfer through convection, which rises the thermal field.

Figure 12 displays the variation in entropy generation $\left(N_{G}\right)$ versus nanofluid volume fraction parameter $(\phi)$, which are stated in terms of $m$. It is obvious that as the $\phi$ increases, so does the $N_{G}$. This is due to the high thermal conductivity $\left(k_{n f}\right)$ of SWCNT/MWCNT nanoparticles. Also, the entropy generation number $N_{G}$ increases with $m$. Figure 13 represents the thermal radiation $(\mathrm{Nr} r)$ impact on the entropy generation number $\left(N_{G}\right)$. An increasing trend in the $N_{r}$ causes an upsurge in the $N_{G}$. The effects of entropy generation number $\left(N_{G}\right)$ versus Hartmann number $(M)$ for electromagnetic SWCNT/MWCNT- $\mathrm{H}_{2} \mathrm{O}$ nanofluids flow are depicted in Figure 14. The increase in $M$ causes an escalation in $N_{G}$. Physically, this means that the increasing estimations of $M$ accelerates the drag force that enhances the dissipation energy, which is the primary cause of irreversibility. Figure 15 shows the Eckert number's $(E c)$ impact on the entropy generation number $\left(N_{G}\right)$. For all considered cases, the $N_{G}$ rises with the rise in $E c$. Figure 16 describes the impacts of $(B i)$, on $\left(N_{G}\right)$. We discover that $N_{G}$ have a minimum value when $B i$ is small, indicating poor convection in the surfaces, and then begin to improve until stability is obtained when $B i \longrightarrow \infty$ (isotherm-surface). All three flow instances exhibit the same behavior. As a result, minimizing convection via boundaries can reduce entropy generation. Figures 17(a) and 17(b) illustrate the effect of $\phi$ on Bejan number $\left(B_{e}\right)$ for electromagnetic SWCNT- $\mathrm{H}_{2} \mathrm{O}$ and MWCNT $-\mathrm{H}_{2} \mathrm{O}$ nanofluids flow, respectively. In both situations, an increase in $\phi$ elevates $B_{e}$. Figure 18 shows the 


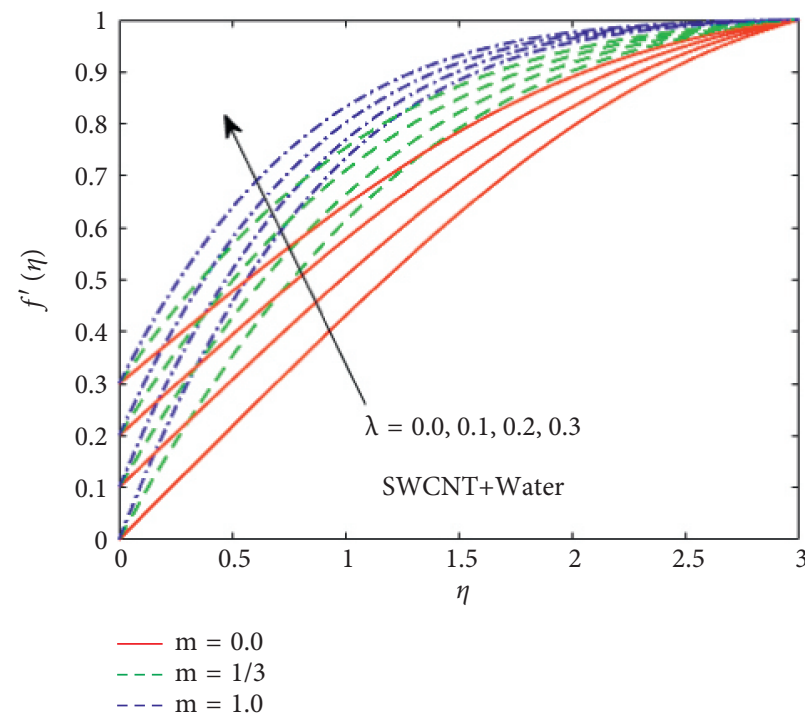

(a)

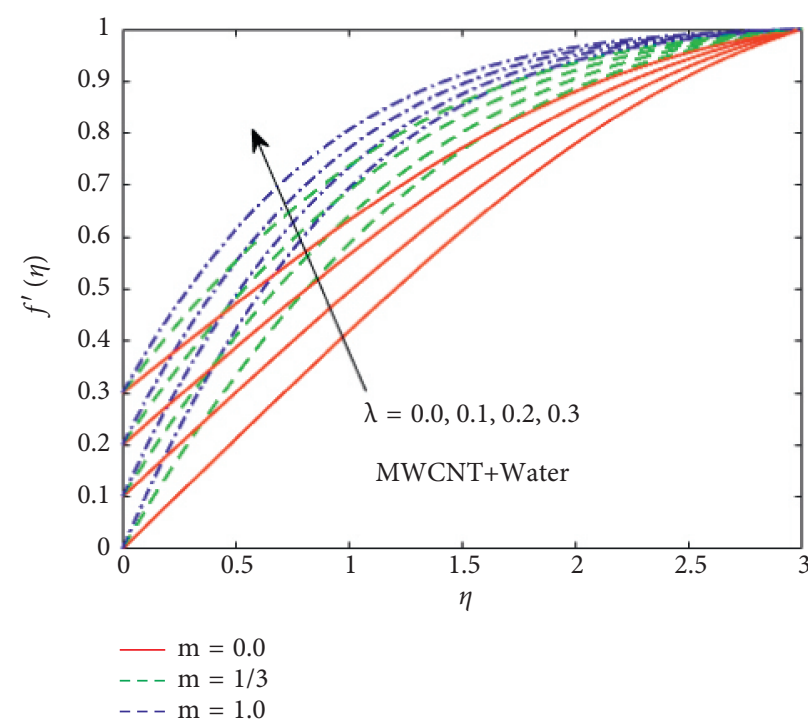

(b)

FIGURE 5: $\lambda$ influence on $f^{\prime}(\eta)$ for (a) SWCNT + water (b) MWCNT + water.

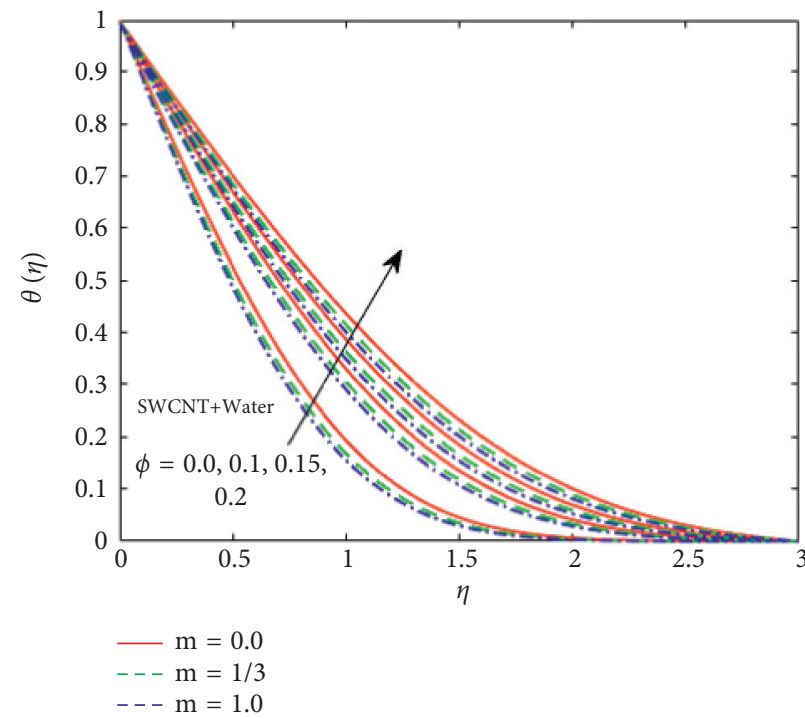

(a)

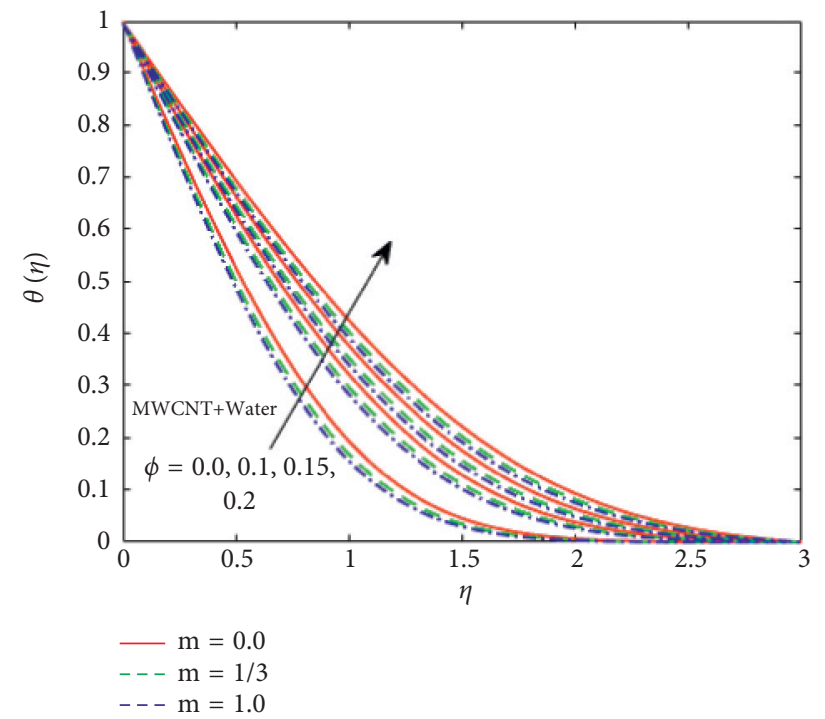

(b)

FIgURE 6: $\phi$ influence on $\theta(\eta)$ for (a) SWCNT + water (b) MWCNT + water.

thermal radiation's effect on the Bejan number $\left(B_{e}\right) . B_{e}$ increases as the radiation parameter $(N r)$ is increased. Greater radiation-absorption inside boundary zone raises system's energy, which causes an increase in $B_{e}$. Figure 19 reveals the influence of the convective surface parameter $(B i)$ on the Bejan number $\left(B_{e}\right)$. When $B i$ is small, we discover that $B_{e}$ has a minimal value. The behavior is consistent across all three flow cases. 

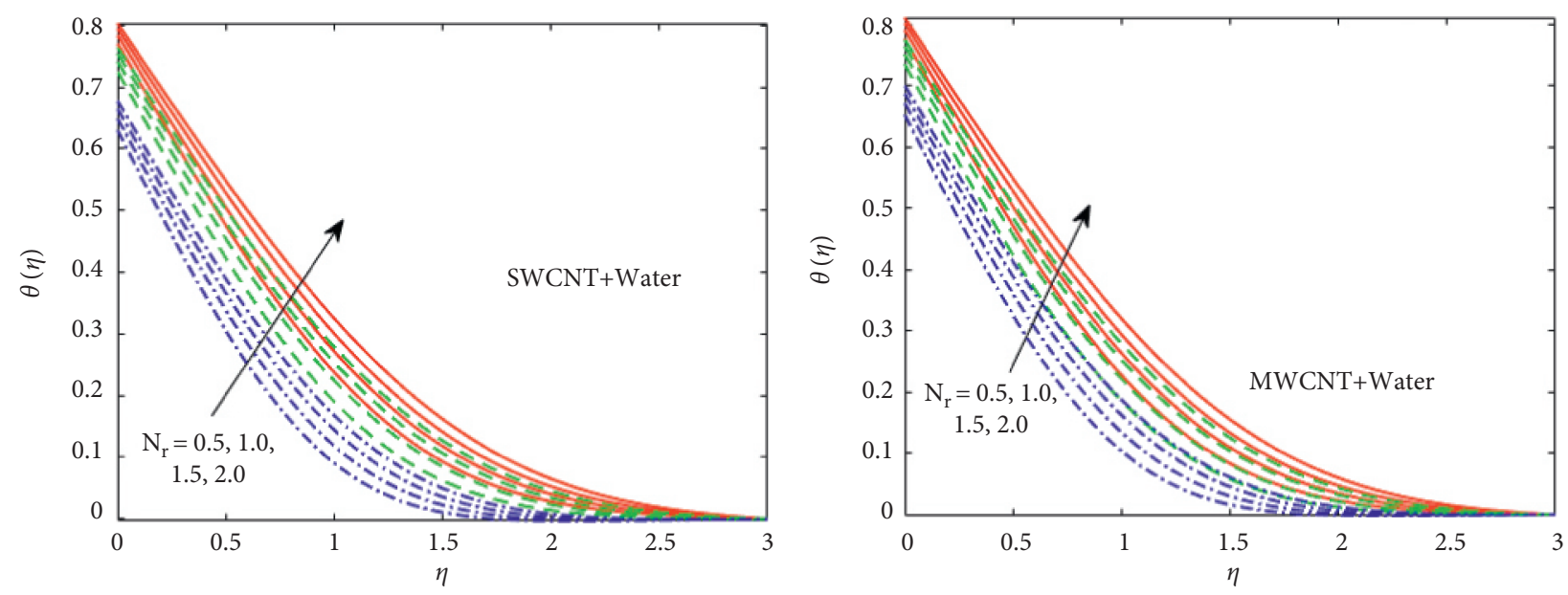

$$
\begin{aligned}
\mathrm{m} & =0.0 \\
--\mathrm{m} & =1 / 3 \\
--\mathrm{m} & =1.0
\end{aligned}
$$

$-\mathrm{m}=0.0$

$--\mathrm{m}=1 / 3$

$---\mathrm{m}=1.0$

(a)

(b)

Figure 7: $N r$ influence on $\theta(\eta)$ for (a) SWCNT + water (b) MWCNT + water.

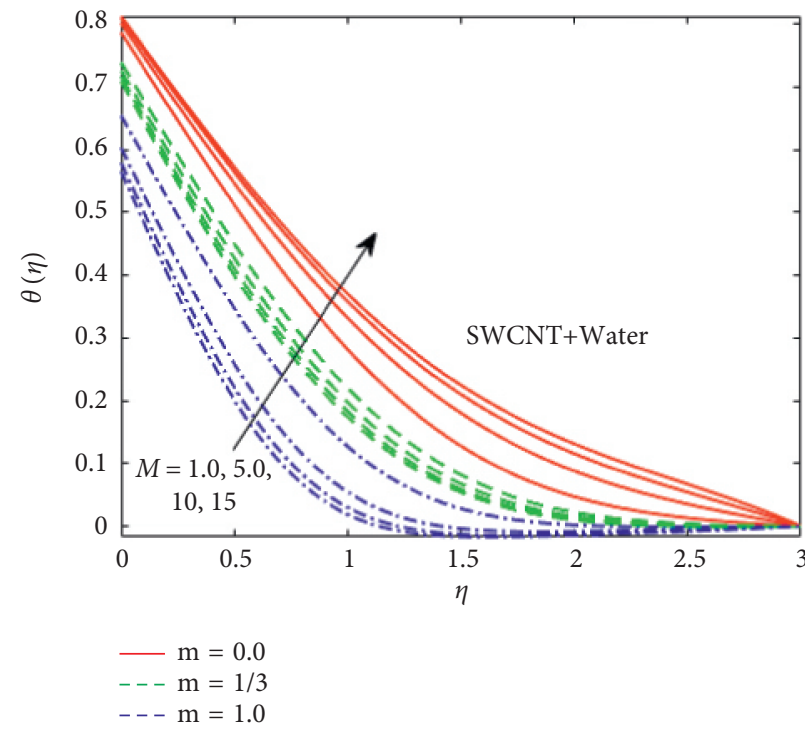

(a)

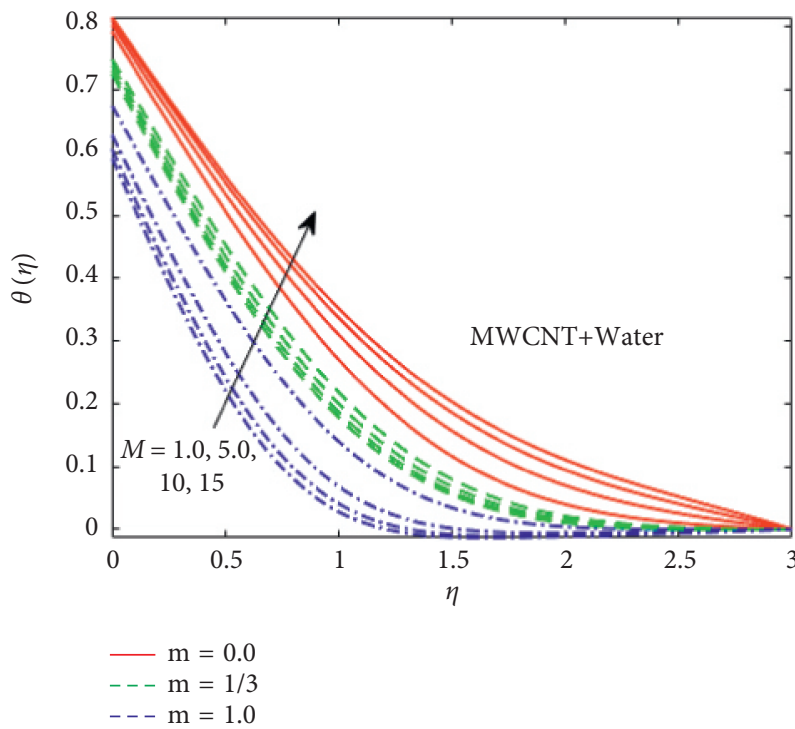

(b)

FIGURE 8: $M$ influence on $\theta(\eta)$ for (a) SWCNT + water (b) MWCNT + water. 


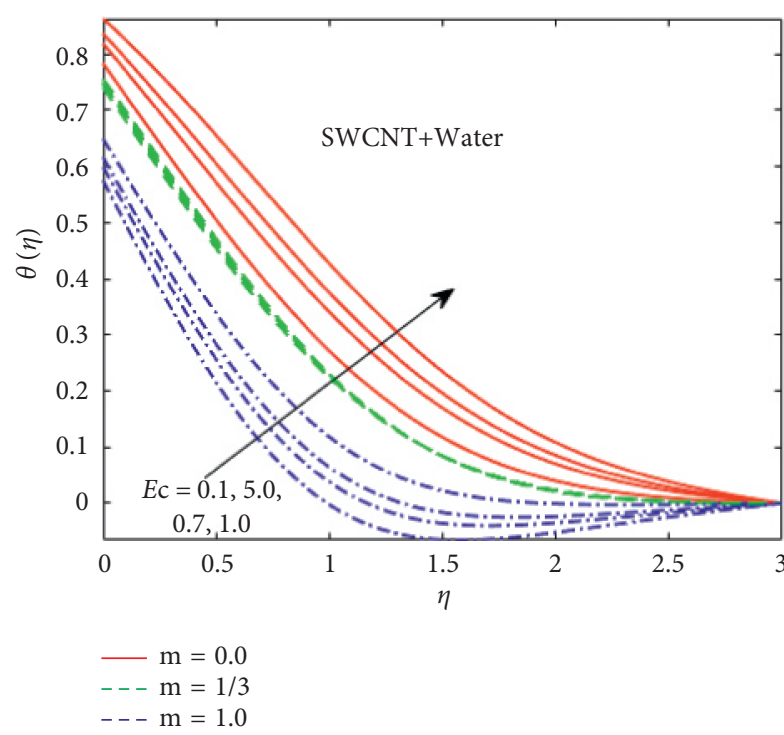

(a)

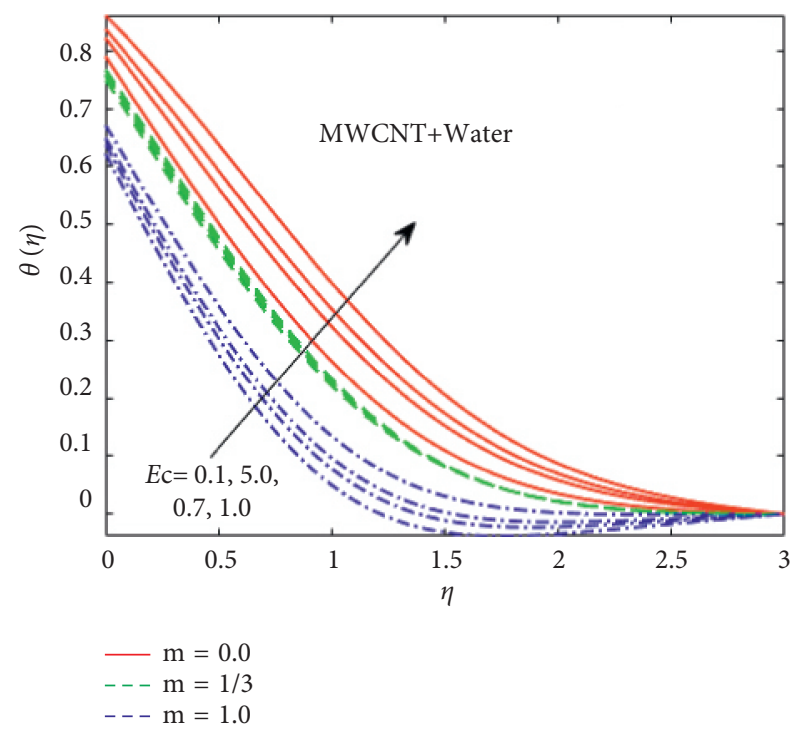

(b)

Figure 9: $E c$ influence on $\theta(\eta)$ for (a) SWCNT + water (b) MWCNT + water.

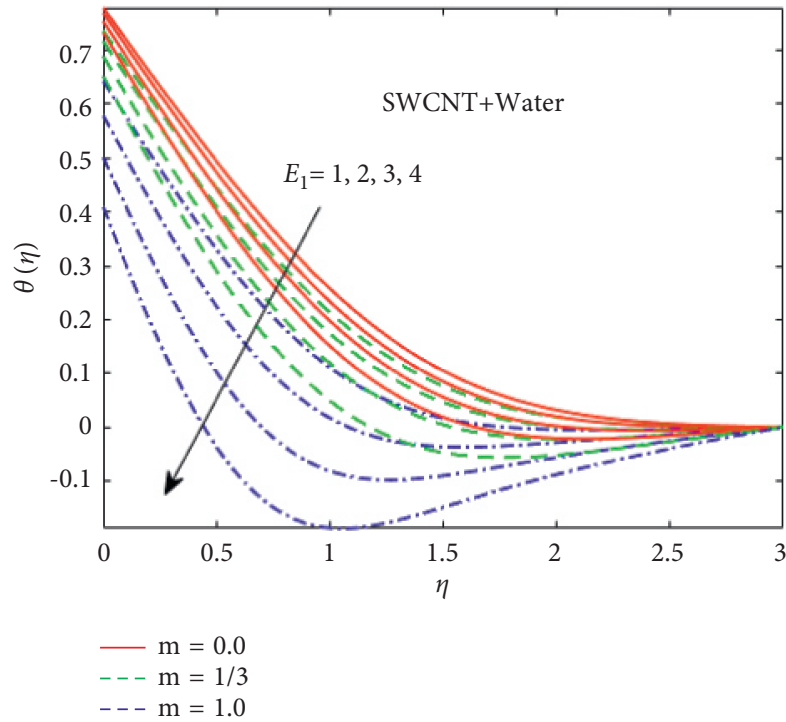

(a)

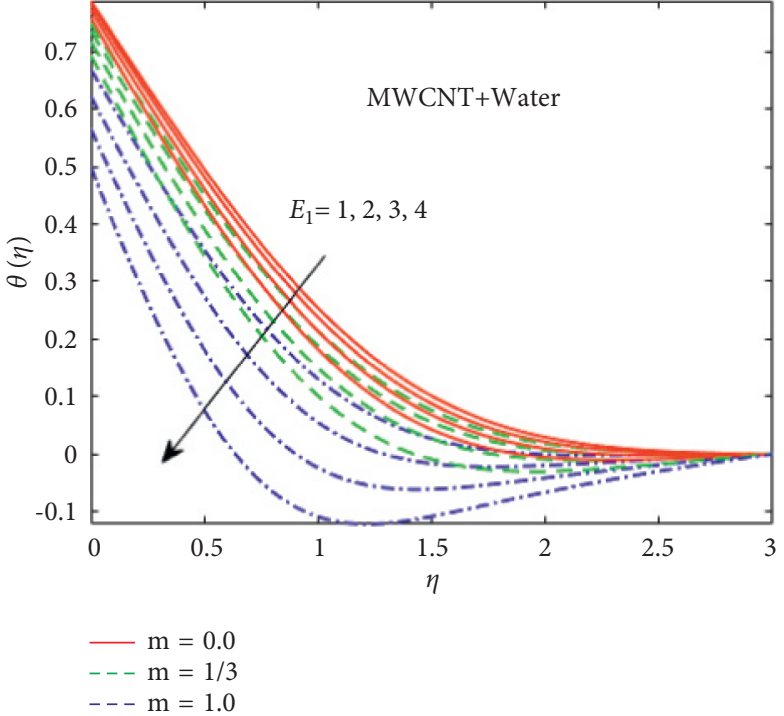

(b)

FIgURE 10: $E_{1}$ influence on $\theta(\eta)$ for (a) SWCNT + water (b) MWCNT + water. 


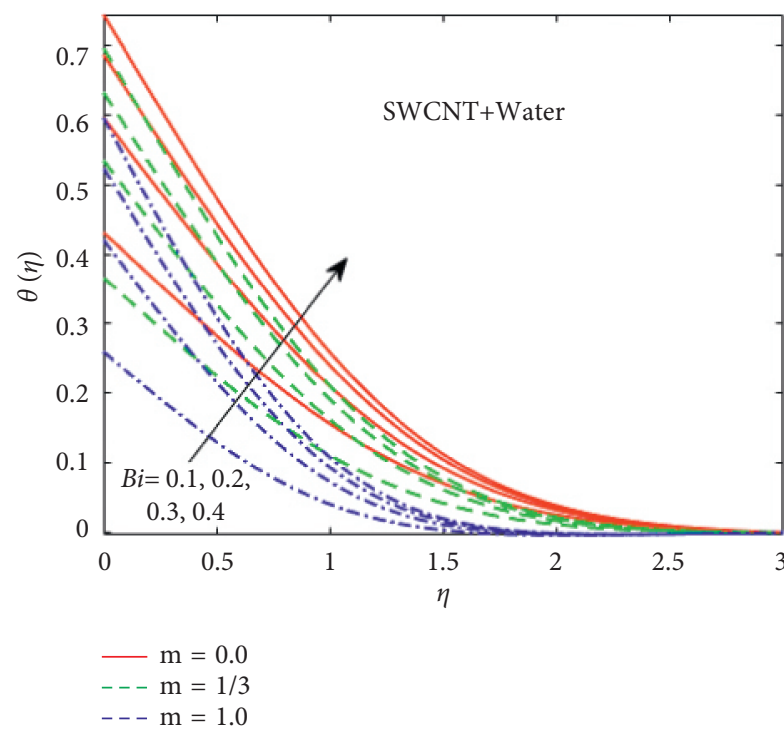

(a)

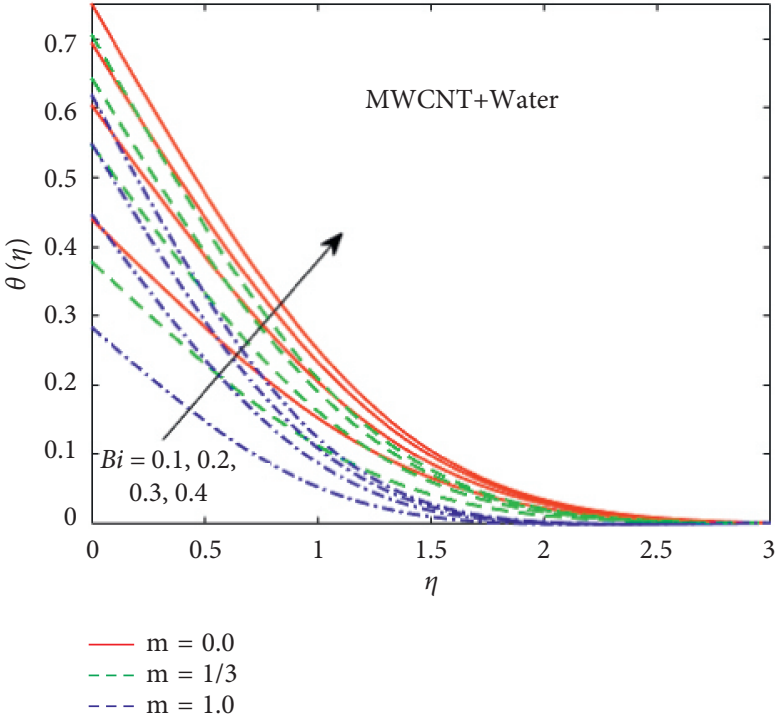

(b)

FIgURE 11: $B i$ influence on $\theta(\eta)$ for (a) SWCNT + water (b) MWCNT + water.

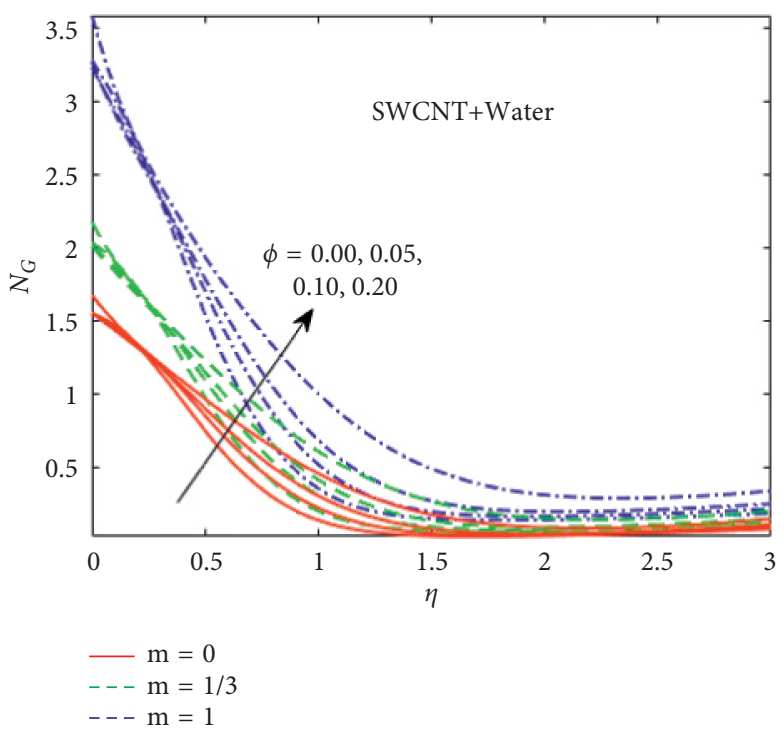

(a)

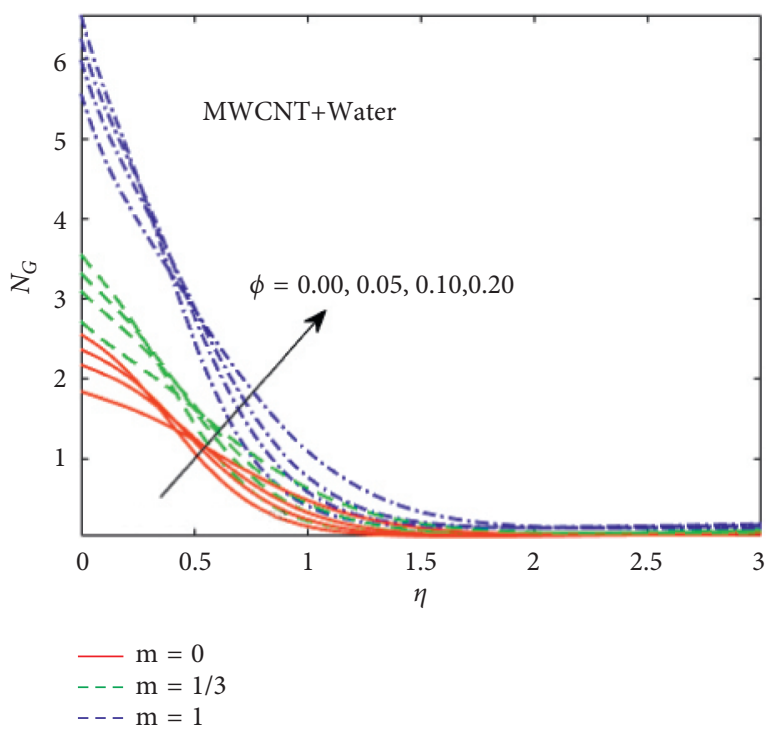

(b)

Figure 12: $\phi$ influence on $N_{G}$ for (a) SWCNT + water (b) MWCNT + water. 


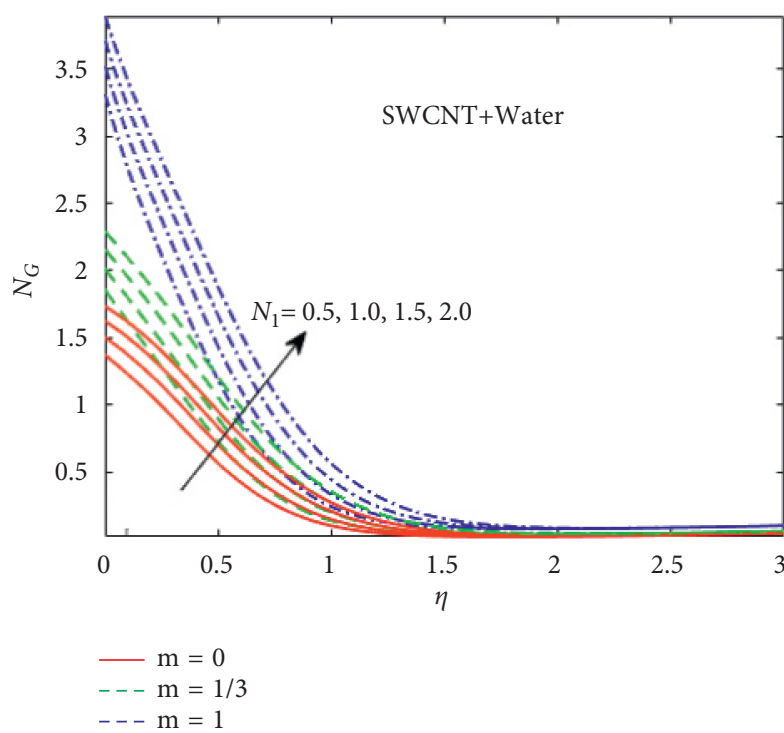

(a)

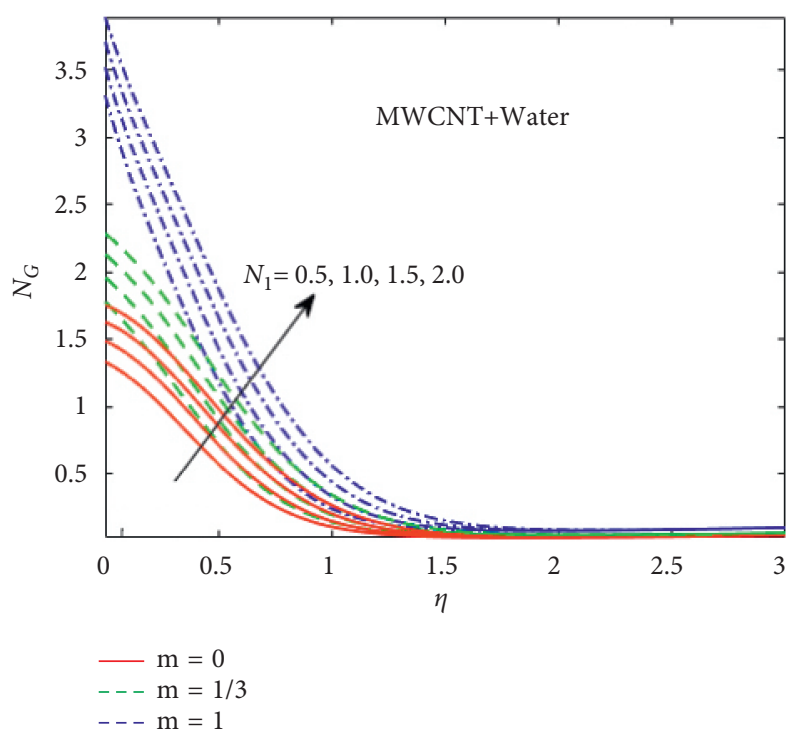

(b)

Figure 13: $N r$ influence on $N_{G}$ for (a) SWCNT + water (b) MWCNT + water.

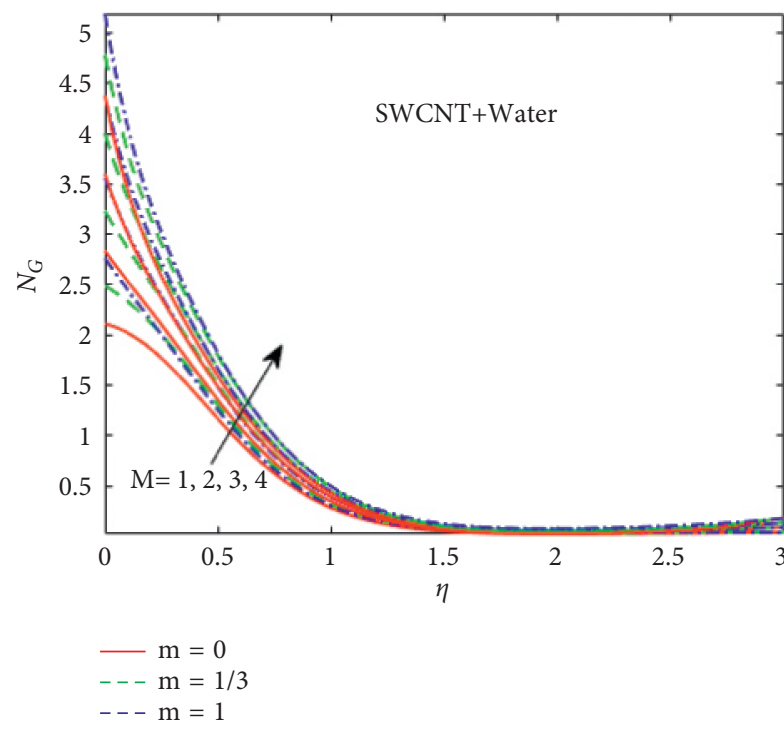

(a)

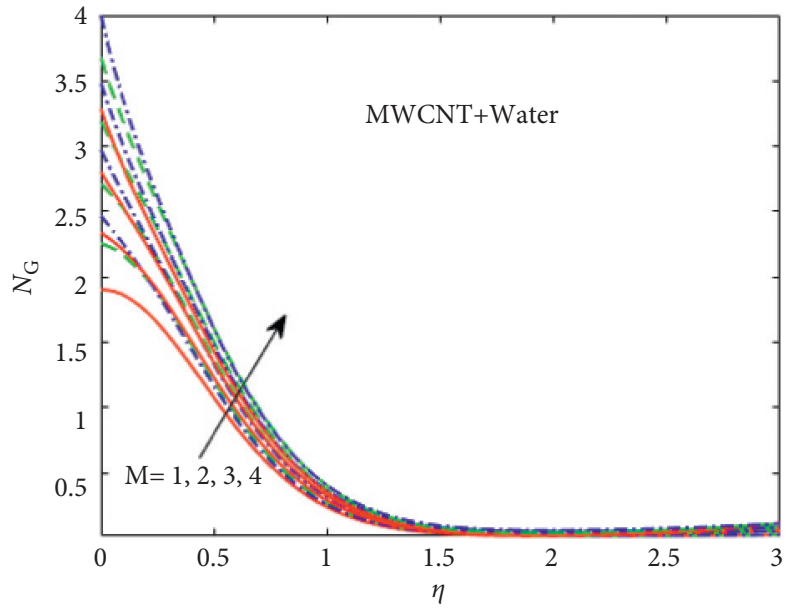

$\begin{aligned} \mathrm{m} & =0 \\ --\mathrm{m} & =1 / 3\end{aligned}$

$-\ldots \mathrm{m}=1$

FIgURE 14: $M$ influence on $N_{G}$ for (a) SWCNT + water (b) MWCNT + water. 


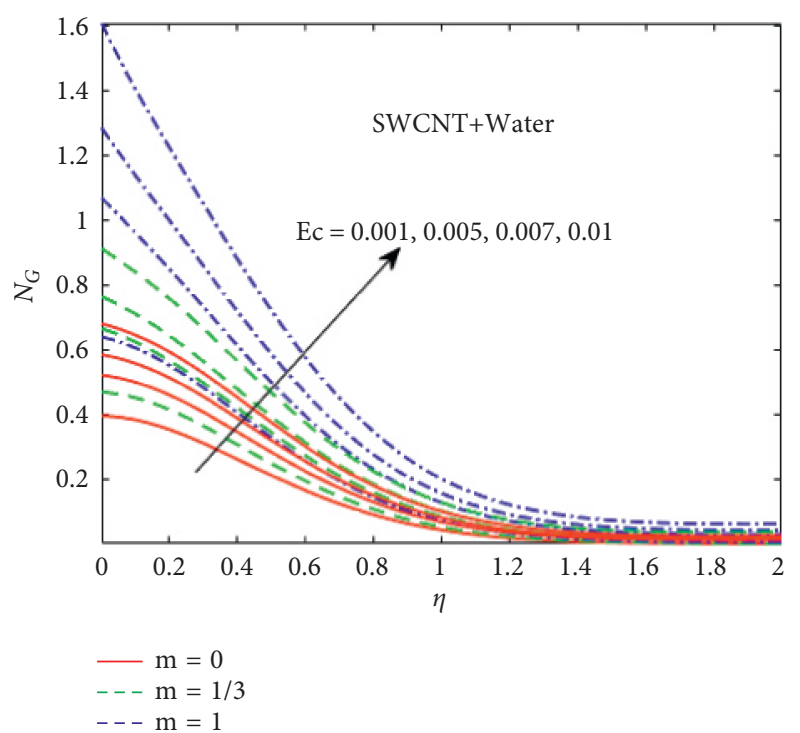

(a)

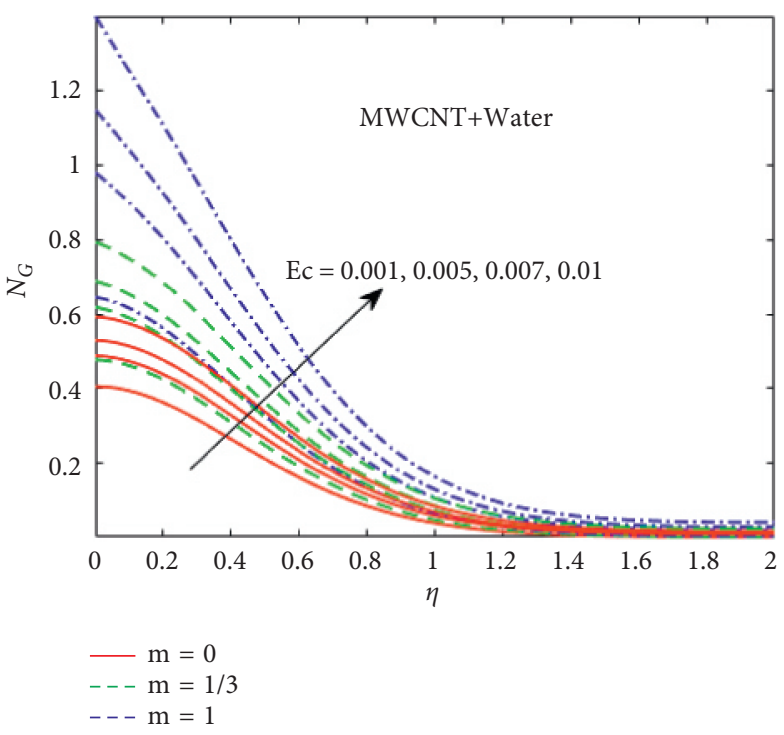

(b)

FIgURE 15: $E c$ influence on $N_{G}$ for (a) SWCNT + water (b) MWCNT + water.

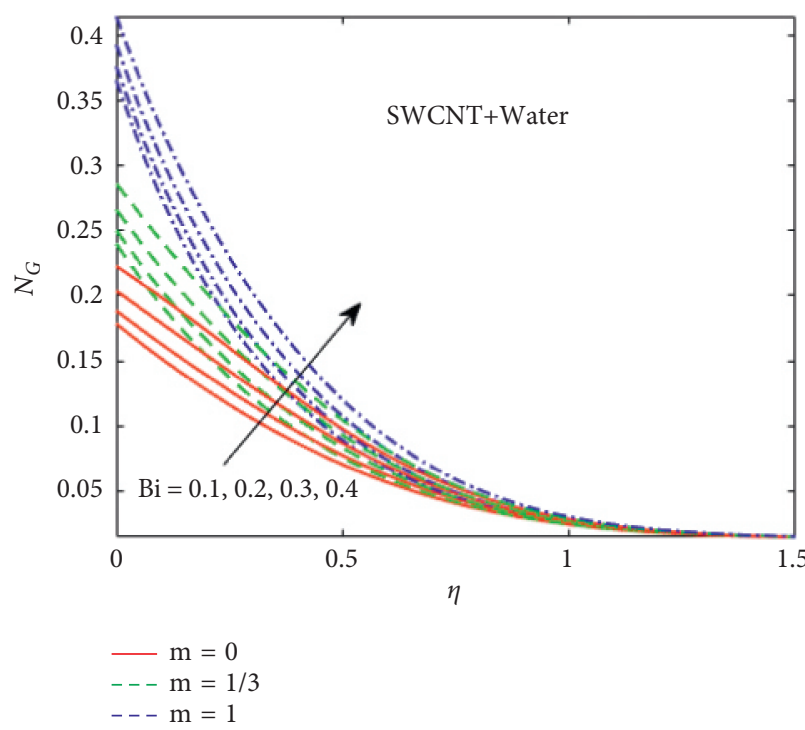

(a)

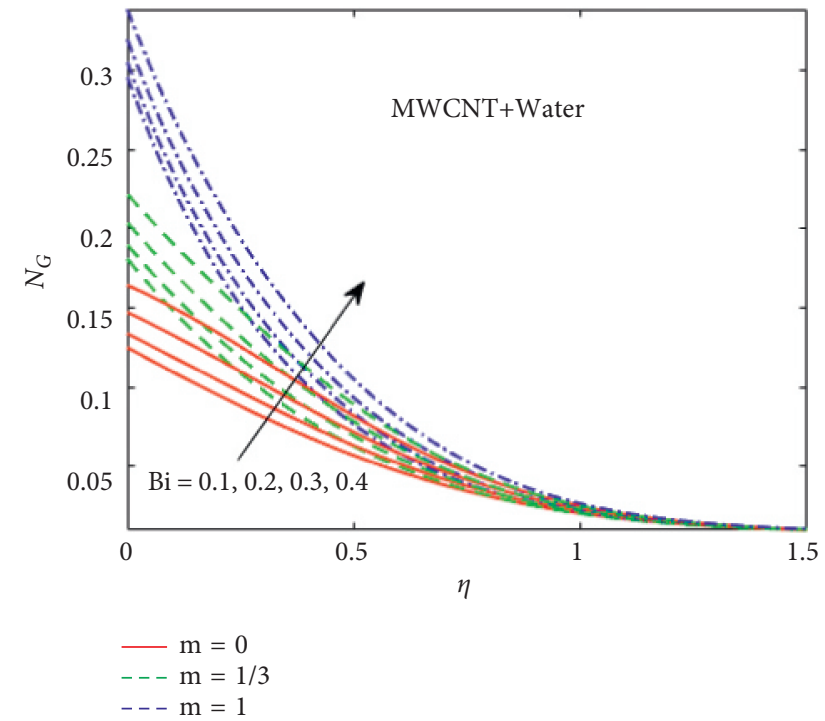

(b)

FIgURE 16: $B i$ influence on $N_{G}$ for (a) SWCNT + water (b) MWCNT + water. 


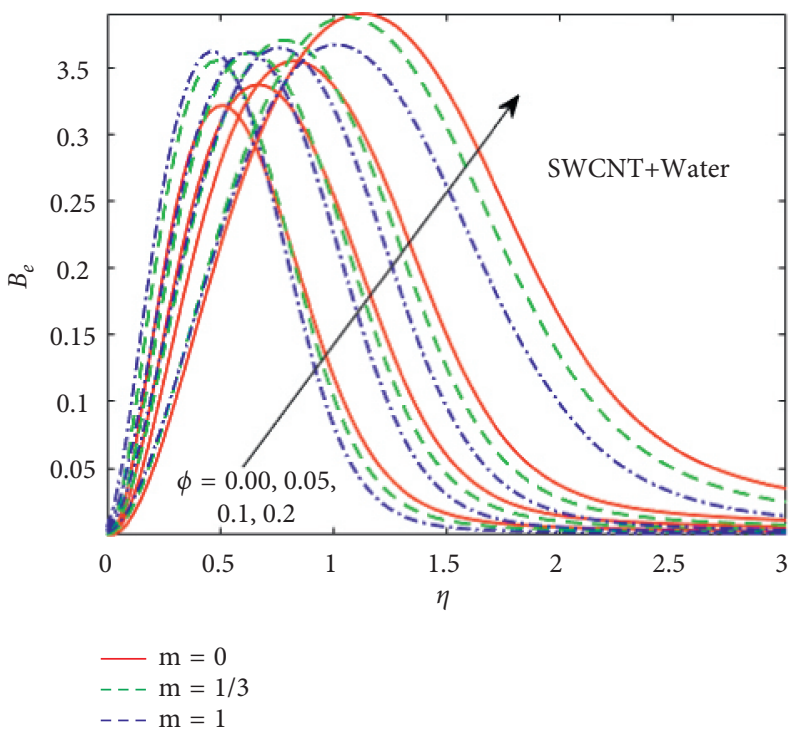

(a)

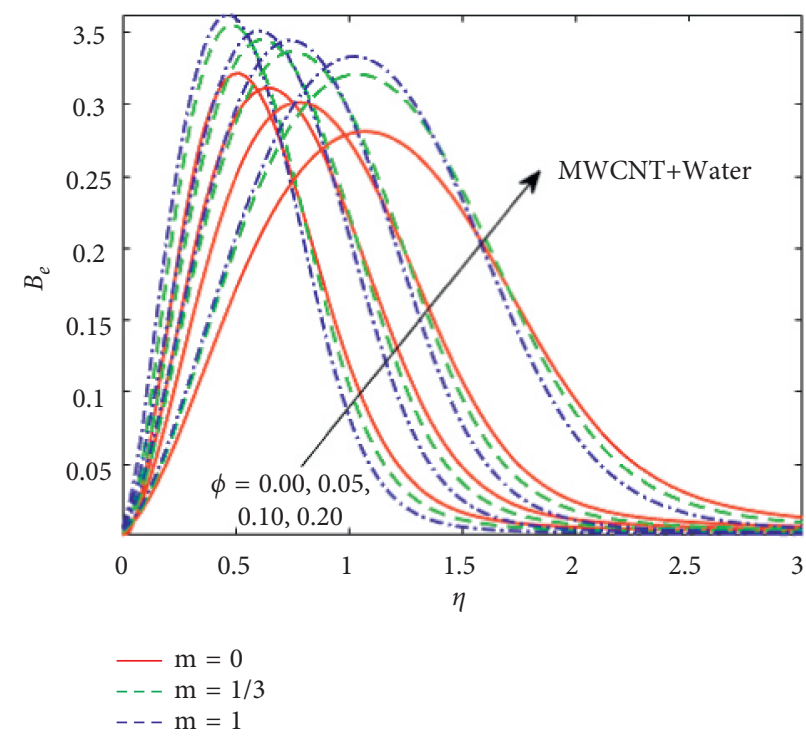

(b)

FIgURE 17: $\phi$ influence on $B_{e}$ for (a) SWCNT + water (b) MWCNT + water.

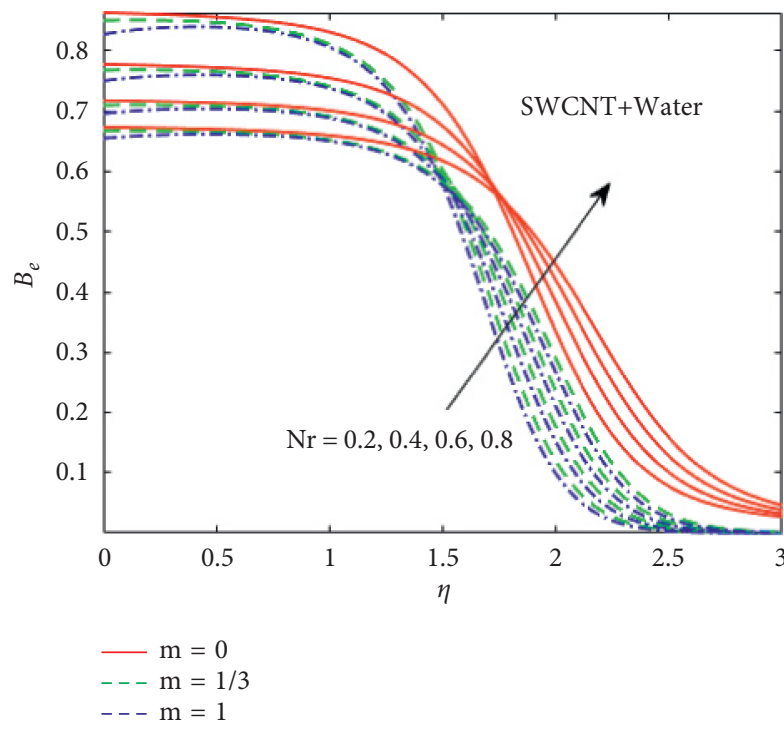

(a)

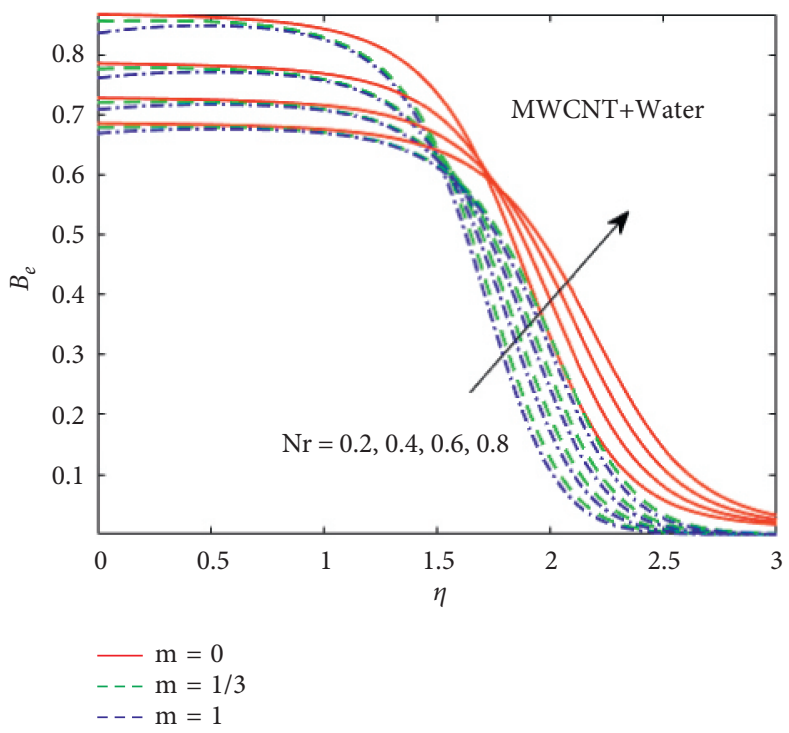

(b)

FIGURE 18: $N r$ influence on $B_{e}$ for (a) SWCNT + water (b) MWCNT + water. 


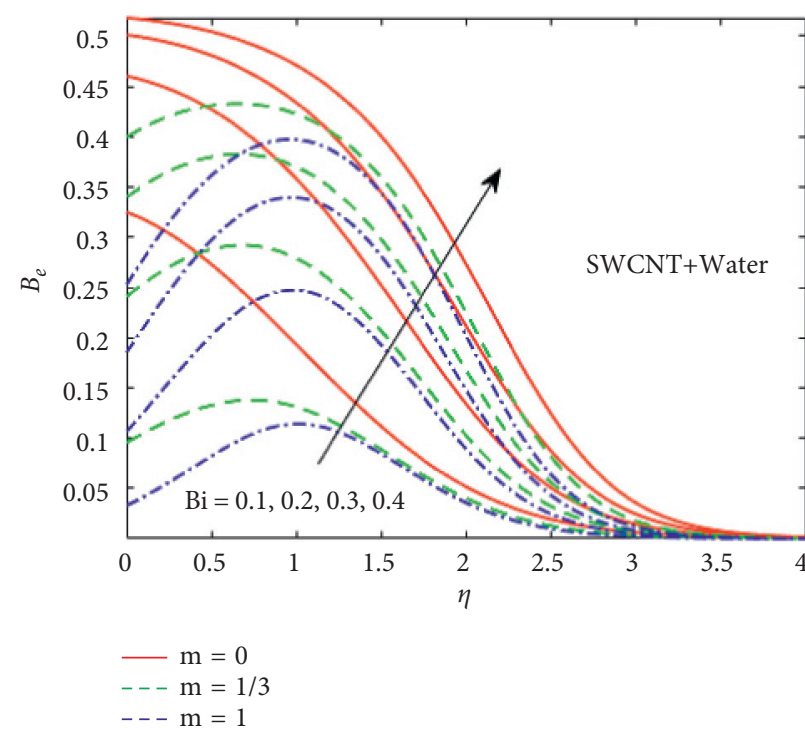

(a)

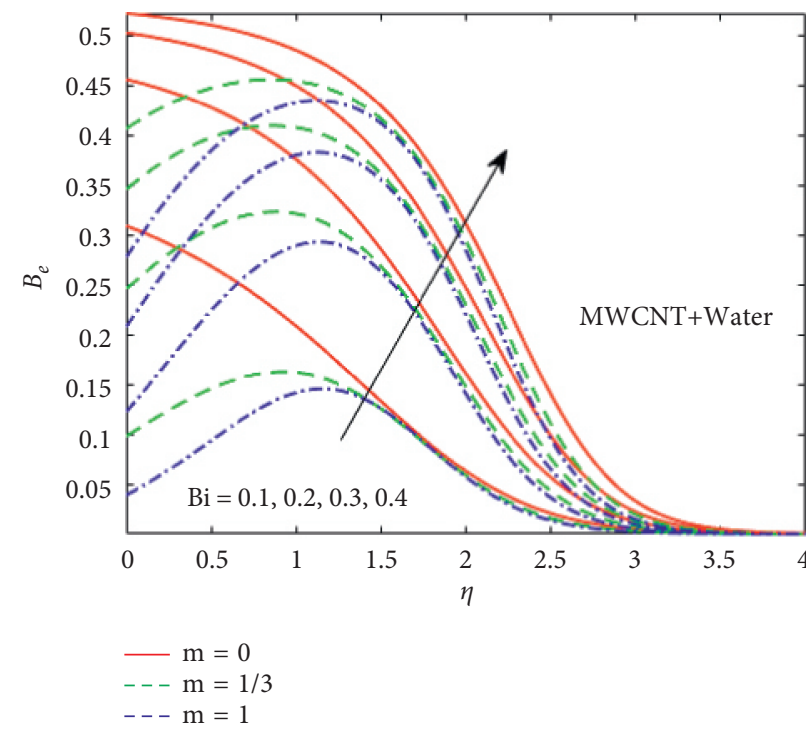

(b)

FIgURE 19: $B i$ influence on $B_{e}$ for (a) SWCNT + water (b) MWCNT + water.

\section{Conclusions}

In this study, we explored numerically the nonsimilar electromagnetic, radiating water/CNTs nanofluid flow across convective heated-moving horizontal/vertical plates and wedge with entropy generation influence. The following are the study's key findings:

(i) Velocity field for electromagnetic SWCNT/ MWCNT- $\mathrm{H}_{2} \mathrm{O}$ nanofluids flow became antithetical to the enhanced values of $\phi$ and $M$

(ii) With increasing estimations of the electric field parameter, the velocity field rises for SWCNT/ MWCNT $-\mathrm{H}_{2} \mathrm{O}$ nanofluids

(iii) Thermal field $\theta(\eta)$ improves with the augmentation of $\phi, N r, E c, B i$, and $M$, while it peters out via incremented $E_{1}$

(iv) Increment in $\lambda, M$ leads to diminution of viscous drag while rise in $\phi, m$, and $E_{1}$ upgrades the viscous drag for both MWCNT/SWCNT-water nanofluid

(v) Nusselt number $(\mathrm{Nu})$ decreases for greater estimations of magnetic parameter, while $N u$ rises for increasing values of $E_{1}, B i, \phi, \lambda$, and $N r$

(vi) The increase in viscous dissipation, measured by the Eckart number $(\mathrm{Ec})$, reduces the heat transfer rate

(vii) Temperature profiles for both SWCNT and MWCNT nanofluids fall as the angle of the wedge increases

(viii) For increasing $\phi, M, B i$, and $E c$, entropy generation in boundary-layer nanofluid flow increases

(ix) Entropy generation can be reduced by limiting convection across boundaries (x) The optimum geometry to minimize entropy generation is a horizontal plate corresponding to the wedge angle $m=0$

(xi) Bejan number $\left(B_{e}\right)$ rises with an increment in $\mathrm{Bi}$ and $\phi$ parameters for both SWCNT and MWCNT

(xii) High radiation absorption within the boundary layer raises the system's internal energy, causing an increase in $N_{G}$ and $B_{e}$

\section{Data Availability}

The data used to support the findings of this study are included within the article.

\section{Conflicts of Interest}

The authors declare that they have no conflicts of interest.

\section{Acknowledgments}

The authors extend their appreciation to the Deanship of Scientific Research at King Khalid University, Abha, Saudi Arabia, for funding this work through research groups program under grant number RGP.2/20/43.

\section{References}

[1] S. Iijima, "Helical microtubules of graphitic carbon," Nature, vol. 354, no. 6348, pp. 56-58, 1991.

[2] R. H. Baughman, A. A. Zakhidov, and W. A. De Heer, "Carbon nanotubes--the route toward applications," Science, vol. 297, no. 5582, pp. 787-792, 2002. 
[3] A. Bianco, K. Kostarelos, and M. Prato, "Applications of carbon nanotubes in drug delivery," Current Opinion in Chemical Biology, vol. 9, no. 6, pp. 674-679, 2005.

[4] M. F. L. De Volder, S. H. Tawfick, R. H. Baughman, and A. J. Hart, "Carbon nanotubes: present and future commercial applications," Science, vol. 339, no. 6119, pp. 535-539, 2013.

[5] N. S. Akbar, Z. H. Khan, and S. Nadeem, "The combined effects of slip and convective boundary conditions on stagnation-point flow of CNT suspended nanofluid over a stretching sheet," Journal of Molecular Liquids, vol. 196, pp. 21-25, 2014.

[6] B. H. Thang, P. H. Khoi, and P. N. Minh, "A modified model for thermal conductivity of carbon nanotube-nanofluids," Physics of Fluids, vol. 27, no. 3, Article ID 032002, 2015.

[7] M. Safaei, G. Ahmadi, M. Goodarzi, A. Kamyar, and S. Kazi, "Boundary layer flow and heat transfer of FMWCNT/water nanofluids over a flat plate," Fluid, vol. 1, no. 4, p. 31, 2016.

[8] A. Shahsavar, M. R. Salimpour, M. Saghafian, and M. B. Shafii, "Effect of magnetic field on thermal conductivity and viscosity of a magnetic nanofluid loaded with carbon nanotubes," Journal of Mechanical Science and Technology, vol. 30, no. 2, pp. 809-815, 2016.

[9] D. Lu, M. Ramzan, S. Ahmad, J. D. Chung, and U. Farooq, "Upshot of binary chemical reaction and activation energy on carbon nanotubes with Cattaneo-Christov heat flux and buoyancy effects," Physics of fluids, vol. 29, no. 12, Article ID 123103, 2017.

[10] R. U. Haq, I. Rashid, and Z. H. Khan, "Effects of aligned magnetic field and CNTs in two different base fluids over a moving slip surface," Journal of Molecular Liquids, vol. 243, pp. 682-688, 2017.

[11] S. Mosayebidorcheh and M. Hatami, "Heat transfer analysis in carbon nanotube-water between rotating disks under thermal radiation conditions," Journal of Molecular Liquids, vol. 240, pp. 258-267, 2017.

[12] Z. Iqbal, E. Azhar, and E. N. Maraj, “Transport phenomena of carbon nanotubes and bioconvection nanoparticles on stagnation point flow in presence of induced magnetic field," Physica E: Low-Dimensional Systems and Nanostructures, vol. 91, pp. 128-135, 2017.

[13] D. Lu, Z. Li, M. Ramzan, A. Shafee, and J. D. Chung, "Unsteady squeezing carbon nanotubes based nano-liquid flow with Cattaneo-Christov heat flux and homogeneous-heterogeneous reactions," Applied Nanoscience, vol. 9, no. 2, pp. 169-178, 2019.

[14] S. Ahmad, S. Nadeem, N. Muhammad, and A. Issakhov, "Radiative SWCNT and MWCNT nanofluid flow of FalknerSkan problem with double stratification," Physica A: Statistical Mechanics and Its Applications, vol. 547, Article ID 124054, 2020.

[15] H. Upreti, A. K. Pandey, M. Kumar, and O. D. Makinde, "Ohmic heating and non-uniform heat source/sink roles on 3D Darcy-f flow of CNTs nanofluids over a stretching surface," Arabian Journal for Science and Engineering, vol. 45, no. 9, pp. 7705-7717, 2020.

[16] A. Bejan, "Second law analysis in heat transfer," Energy, vol. 5, no. 8-9, pp. 720-732, 1980.

[17] F. A. Soomro, Z. H. Khan, and Q. Zhang, "Numerical study of entropy generation in MHD water-based carbon nanotubes along an inclined permeable surface," The European Physical Journal Plus, vol. 132, no. 10, pp. 1-12, 2017.

[18] M. Ishaq, G. Ali, Z. Shah, S. Islam, and S. Muhammad, "Entropy generation on nanofluid thin film flow of e-powell fluid with thermal radiation and MHD effect on an unsteady porous stretching sheet," Entropy, vol. 20, no. 6, p. 412, 2018.

[19] U. Farooq, M. Afridi, M. Qasim, and D. Lu, "Transpiration and viscous dissipation effects on entropy generation in hybrid nanofluid flow over a nonlinear radially stretching disk," Entropy, vol. 20, no. 9, p. 668, 2018.

[20] M. Suleman, M. Ramzan, M. Zulfiqar et al., "Entropy analysis of 3D non-Newtonian MHD nanofluid flow with nonlinear thermal radiation past over exponential stretched surface," Entropy, vol. 20, no. 12, p. 930, 2018.

[21] M. Sheikholeslami, M. B. Gerdroodbary, R. Moradi, A. Shafee, and Z. Li, "Application of Neural Network for estimation of heat transfer treatment of Al2O3-H2O nanofluid through a channel," Computer Methods in Applied Mechanics and Engineering, vol. 344, pp. 1-12, 2019.

[22] N. Khan, Z. Shah, S. Islam, I. Khan, T. Alkanhal, and I. Tlili, "Entropy generation in MHD mixed convection non-Newtonian second-grade nanoliquid thin film flow through a porous medium with chemical reaction and stratification," Entropy, vol. 21, no. 2, p. 139, 2019.

[23] N. S. Khan, S. Zuhra, and Q. Shah, "Entropy generation in two phase model for simulating flow and heat transfer of carbon nanotubes between rotating stretchable disks with cubic autocatalysis chemical reaction," Applied Nanoscience, vol. 9, no. 8, pp. 1797-1822, 2019.

[24] A. Dawar, Z. Shah, W. Khan, M. Idrees, and S. Islam, "Unsteady squeezing flow of magnetohydrodynamic carbon nanotube nanofluid in rotating channels with entropy generation and viscous dissipation," Advances in Mechanical Engineering, vol. 11, no. 1, Article ID 1687814018823100, 2019.

[25] M. Ramzan, M. Mohammad, F. Howari, and J. D. Chung, "Entropy analysis of carbon nanotubes based nanofluid flow past a vertical cone with thermal radiation," Entropy, vol. 21, no. 7, p. 642, 2019.

[26] U. Farooq, S. Munir, F. Malik, B. Ahmad, and D. Lu, "Aspects of entropy generation for the non-similar three-dimensional bioconvection flow of nanofluids," AIP Advances, vol. 10, no. 7, Article ID 075110, 2020.

[27] F. E. Alsaadi, K. Muhammad, T. Hayat, A. Alsaedi, and S. Asghar, "Numerical study of melting effect with entropy generation minimization in flow of carbon nanotubes," Journal of Thermal Analysis and Calorimetry, vol. 140, no. 1, pp. 321-329, 2020.

[28] K. Singh, A. K. Pandey, and M. Kumar, "Entropy generation impact on flow of micropolar fluid via an inclined channel with non-uniform heat source and variable fluid properties," International Journal of Algorithms, Computing and Mathematics, vol. 6, pp. 1-12, 2020.

[29] A. K. Verma, A. K. Gautam, K. Bhattacharyya, and I. Pop, "Entropy generation analysis of Falkner-Skan flow of Maxwell nanofluid in porous medium with temperature-dependent viscosity," Pramana, vol. 95, no. 2, pp. 1-16, 2021.

[30] A. Ishak, N. A. Yacob, and N. Bachok, "Radiation effects on the thermal boundary layer flow over a moving plate with convective boundary condition," Meccanica, vol. 46, no. 4, pp. 795-801, 2011.

[31] N. S. Akbar, S. Nadeem, R. Ul Haq, and Z. H. Khan, "Radiation effects on MHD stagnation point flow of nano fluid towards a stretching surface with convective boundary condition," Chinese Journal of Aeronautics, vol. 26, no. 6, pp. 1389-1397, 2013.

[32] S. Nadeem and R. U. Haq, "Effect of thermal radiation for megnetohydrodynamic boundary layer flow of a nanofluid past a stretching sheet with convective boundary conditions," 
Journal of Computational and Theoretical Nanoscience, vol. 11, no. 1, pp. 32-40, 2014.

[33] A. A. Delouei, M. Nazari, M. H. Kayhani, and S. Succi, "Immersed boundary - thermal lattice Boltzmann methods for non-Newtonian flows over a heated cylinder: a comparative study," Communications in Computational Physics, vol. 18, no. 2, pp. 489-515, 2015.

[34] I. S. Oyelakin, S. Mondal, and P. Sibanda, "Unsteady Casson nanofluid flow over a stretching sheet with thermal radiation, convective and slip boundary conditions," Alexandria Engineering Journal, vol. 55, no. 2, pp. 1025-1035, 2016.

[35] A. Zaib, A. Banerjee, and K. Bhattacharyya, "Impact of homogeneous-heterogeneous reactions on mixed convection flow of a copper-water nanofluid past a permeable shrinking cylinder with thermal radiation," Proceedings of the Institution of Mechanical Engineers - Part E: Journal of Process Mechanical Engineering, vol. 232, no. 5, pp. 566-578, 2018.

[36] K. Jyothi, P. Sudarsana Reddy, and M. Suryanarayana Reddy, "Influence of magnetic field and thermal radiation on convective flow of SWCNTs-water and MWCNTs-water nanofluid between rotating stretchable disks with convective boundary conditions," Powder Technology, vol. 331, pp. 326-337, 2018.

[37] S. R. R. Reddy, P. Bala Anki Reddy, and K. Bhattacharyya, "Effect of nonlinear thermal radiation on 3D magneto slip flow of Eyring-Powell nanofluid flow over a slendering sheet with binary chemical reaction and Arrhenius activation energy," Advanced Powder Technology, vol. 30, no. 12, pp. 3203-3213, 2019.

[38] R. S. Saif, T. Muhammad, H. Sadia, and R. Ellahi, "Boundary layer flow due to a nonlinear stretching curved surface with convective boundary condition and homogeneous-heterogeneous reactions," Physica A: Statistical Mechanics and Its Applications, vol. 551, Article ID 123996, 2020.

[39] A. Raees, U. Farooq, M. Hussain, W. A. Khan, and F. B. Farooq, "Non-similar mixed convection analysis for magnetic flow of second-grade nanofluid over a vertically stretching sheet," Communications in Theoretical Physics, vol. 73, no. 6, Article ID 065801, 2021.

[40] U. Farooq, M. Hussain, M. A. Ijaz, W. A. Khan, and F. Bashir Farooq, "Impact of non-similar modeling on Darcy-Forchheimer-Brinkman model for forced convection of Casson nano-fluid in non-Darcy porous media," International Communications in Heat and Mass Transfer, vol. 125, Article ID 105312, 2021.

[41] H. Berrehal, F. Mabood, and O. D. Makinde, "Entropy-optimized radiating water/FCNTs nanofluid boundary-layer flow with convective condition," The European Physical Journal Plus, vol. 135, no. 7, pp. 1-21, 2020.

[42] E. M. Sparrow and H. S. Yu, "Local non-similarity thermal boundary-layer solutions," Journal of Heat Transfer, vol. 93, no. 4, pp. 328-334, 1971.

[43] W. N. I. N. Noranuar, A. Q. Mohamad, S. Shafie, I. Khan, M. R. Ilias, and L. Y. Jiann, Analysis of Heat Transfer in Noncoaxial Rotation of Newtonian Carbon Nanofluid Flow with Magnetohydrodynamics and Porosity Effects, IntechOpen, London, UK, 2021.

[44] K. A. Yih, "Uniform suction/blowing effect on forced convection about a wedge: uniform heat flux," Acta Mechanica, vol. 128, no. 3, pp. 173-181, 1998.

[45] N. A. Yacob, A. Ishak, and I. Pop, "Falkner-Skan problem for a static or moving wedge in nanofluids," International Journal of Thermal Sciences, vol. 50, no. 2, pp. 133-139, 2011. 